\title{
Second harmonic generation and birefringence of some ternary pnictide semiconductors
}

\author{
Sergey N. Rashkeev, Sukit Limpijumnong and Walter R. L. Lambrecht \\ Department of Physics, Case Western Reserve University, Cleveland, OH 44106-7079
}

(March 12, 2021)

\begin{abstract}
A first-principles study of the birefringence and the frequency dependent second harmonic generation (SHG) coefficients of the ternary pnictide semiconductors with formula $\mathrm{ABC}_{2}(\mathrm{~A}=\mathrm{Zn}$, $\mathrm{Cd} ; \mathrm{B}=\mathrm{Si}, \mathrm{Ge} ; \mathrm{C}=\mathrm{As}, \mathrm{P})$ with the chalcopyrite structures was carried out. It uses a recently developed computational approach based on the self-consistent linear muffin-tin orbital (LMTO) band-structure method, which is applied using the local density approximation to density functional theory with a simple a-posteriori gap correction. The susceptibilies are obtained in the independent particle approximation, i.e., without local field and excitonic effects. The zero frequency limits of $\chi_{123}^{(2)}$ were found to be in reasonable agreement with available experimental data for all the considered materials. We found that substitution of $\mathrm{P}$ by As, Si by Ge, and $\mathrm{Zn}$ by $\mathrm{Cd}$ is favorable to get a higher value of $\chi^{(2)}(0)$. However, the anomalously high value of the zero frequency SHG in $\mathrm{CdGeAs}_{2}$ (the material with the most favorable combination of $\mathrm{A}, \mathrm{B}$, and $\mathrm{C}$ ) is rather exceptional than typical for this group of compounds. An analysis of the different contributions in the frequency dependent SHG spectra shows that this value appears as a result of a very small interband term in the zero frequency limit which cannot compensate the large intraband contribution as it happens in most of the other materials of this class. The smallness of the interband term in $\mathrm{CdGeAs}_{2}$ is a result of a very delicate balance between different interband transitions, any of which can give positive or negative contribution in SHG. While we find the empirical observation that a smaller value of the gap is strongly correlated with a larger value of the SHG to be generally true when comparing various element substitutions within this family in a qualitative sense, simple inverse power scaling laws between gaps and $\chi^{(2)}$ values are not supported by our results. The case of $\mathrm{CdGeAs}_{2}$ clearly shows that this is an oversimplification and that for reliable predictions of trends of SHG coefficients one has to study the interplay between different terms which contribute in SHG. We have also studied the relation between $\chi^{(2)}$ and the chalcopyrite crystal structure by considering some of these materials in an alternative layered zincblende type latttice. We find that the (001) oriented $1+1$ superlattice structure has significantly lower gaps than the chalcopyrite and correspondingly higher $\chi^{(2)}$. However, this smaller gap structure is characterized by a large alternatingly compressive and tensile lateral strain in the layers, which makes it unfavorable. We also find that the distortions from the ideal chalcopyrite tend to increase the gap and decrease both the inter- and intraband contributions to $\chi^{(2)}$, but the net value of $\chi^{(2)}$ is only slightly changed in most cases. These effects are larger for the $\mathrm{Cd}$ compounds than for the Zn-compounds. As far as the birefringence is concerned, we find our calculations for $\mathrm{ZnGeP}_{2}$ and $\mathrm{CdGeAs}_{2}$ to be in fair agreement with experiment (discrepancies being rather constant and of order $10 \%$ ) in the frequency range corresponding to the middle of the gap but to deviate from the data when the absorption edges of the band gap at high energy and the phonon absorption bands at low energy are approached. It is presently not clear whether this reflects a deficiency in the theory or imperfections in the samples.
\end{abstract}

PACS numbers: 42.65.Ky, 78.20.Bh

\section{INTRODUCTION}

Ternary chalcopyrites are promising for optical frequency conversion applications in solid state laser systems, such as optic parametric oscillators (OPO) and frequency doubling devices [1]. Zinc germanium diphosphide $\left(\mathrm{ZnGeP}_{2}\right)$ is an excellent nonlinear optical material which exhibits good optical transparency over the 0.7-12 $\mu \mathrm{m}$ wavelength region. This uniaxial crystal has positive birefringence, and its conventional conversion efficiency, or figure of merit $\left(\mathrm{FOM}=\left(\chi^{(2)}\right)^{2} / 4 n^{3}\right.$ where $n$ is the index of refraction) exceeds that of $\mathrm{LiNbO}_{3}$, a presently commonly used material, by an order of magnitide. It is therefore, a very good candidate to producing tunable laser output in the near infrared [2 6]. $\mathrm{CdGeAs}_{2}$ has even larger $\chi^{(2)}$ and FOM. To the best of our knowledge this material has the highest nonlinear optical coefficient in the class of phase-matchable compounds [7] 10]. Recent crystal growth technologies have made great progress towards growth of large crystals of both $\mathrm{ZnGeP}_{2}$ and $\mathrm{CdGeAs}_{2}$ with improved optical quality. These two materials, however, are part of a larger family of II-IV- $V_{2}$ compounds and although the crystal growth perfection 
of these has not been pursued to the extent of the above two, it is of interest to ask whether other materials in this class, or alloys between them might offer increased flexibility in terms of combining high $\chi^{(2)}$ with other ranges of transparency and/or non-critical phase-matching. More generally, it is of interest to understand the origin of the high $\chi^{(2)}$ and the degree of birefringence in these materials as well as to study the trends within this family of compounds.

In the present paper we systematically study the electronic structure and optical properties of a class of ternary pnictides with formula $\mathrm{ABC}_{2}(\mathrm{~A}=\mathrm{Zn}, \mathrm{Cd} ; \mathrm{B}=\mathrm{Si}, \mathrm{Ge} ; \mathrm{C}=\mathrm{As}, \mathrm{P})$ which are crystallizing in the chalcopyrite structure. The measured SHG coefficients have been reported for some of these materials, and their values are generally rather high (at least not much lower than the SHG in GaAs) [10,11]. Our main purpose is to understand the trends in this family of materials and to check whether simple schemes for extrapolation (e.g., based on inverse power relations to the band gap) to other compounds in this family are valid. The choice of A, B, and C atoms allows us to consider the trends in the group-II, group-IV, and group-V atoms separately in this class of materials which has the general formula II-IV- $\mathrm{V}_{2}$. We also note that some of these materials have direct gaps while others have indirect or pseudodirect gaps. Thus, we can investigate whether there is any relation between the type of the gap and the $\chi^{(2)}$ values. In fact, we find little evidence for such a correlation. This result could be expected from the fact that $\chi^{(2)}$ depends on a Brillouin zone average of interband vertical optical transitions and is, therefore, little affected by the bands at a few particular k- points determining the band edges.

Typically, one indeed finds smaller band gap materials to have higher $\chi^{(2)}$ [12]. It is expected from the basic expressions for the NLO response functions [13, 14] and from empirical observations that NLO susceptibilities scale roughly inversely with some power of the interband energy differences, and, therefore, with the minimum gaps if these dominate the response. However, we show that the situation is not that straightforward, and the real value of the zero frequency limit of SHG is a result of a very delicate balance between different contributions to nonlinear optical response, e.g., the intraband and interband contributions. It turns out that $\mathrm{CdGeAs}_{2}$ is a rather exceptional material in this context.

A second purpose is to investigate whether or not the chalcopyrite structure plays a special role in leading to high $\chi^{(2)}$ values. We can test the effect of crystal structure at least qualitatively by considering an alternative crystal structure corresponding to the same overall chemical formula or stoichiometry. To this end we consider a (001) oriented $1+1$ superlattice structure and compare its band structure, total energy, and $\chi^{(2)}$ with that of chalcopyrite for $\mathrm{ZnGeP}_{2}$. We note that this particular structure still maintains the local tetrahedral environment of the chalcopyrite corresponding to each anion being surrounded by two cations of each type. Our results show that the alternative structure considered generally has a much lower band gap and hence higher $\chi^{(2)}$. However, we find that this is related to the occurence of alternatingly compressive and tensile biaxial strain in the layers which make the structure unstable. Thus, we do not think that this alternative structure gives a promise for further increases of $\chi^{(2)}$.

The chalcopyrite structure is typically accompanied by a distortion from the ideal $c / a$ ratio one would obtain by simply substituting II and IV atoms in the cubic zincblende structure in the appropriate ordering. It is thus also of interest to investigate whether this has an important effect on $\chi^{(2)}$. We find that these effects are significantly larger for the $\mathrm{Cd}$ than for the $\mathrm{Zn}$ compounds which are closer to the ideal structure. While the gaps are generally increased and hence, both intra- and interband contributions are significantly decreased, the net value of $\chi^{(2)}$ is only slightly changed as a result of the compensation noted above.

The computational approach used in the present investigation was described in our previous paper [15] and successfully applied to $\mathrm{SiC}$ polytypes [16] and GaN/AlN superlattices [17].

The rest of the paper is organized as follows. In Section II we describe briefly the necessary details of our computational approach. In Section III we present our calculated results for the electronic band structures, static second harmonic generation coefficients and birefringence and address the various questions raised in this introduction. We analyze the results in terms of the decomposition of the frequency dependent $\operatorname{Im} \chi^{(2)}(-2 \omega, \omega, \omega)$ function on intraand interband terms as defined in Refs. [13] and 14]. A summary of the main conclusions of this work is given in Sec. IV.

\section{COMPUTATIONAL METHOD}

Our calculations are based on the linear muffin-tin orbitals method (LMTO) 18,19] within the atomic sphere approximation (ASA). This method is quite efficient mainly because it employs a rather small basis set. It allows to easily deal with systems containing a large number of atoms per unit cell while maintaining a sufficiently large number of k-points so as to ensure converged Brillouin zone (BZ) integrations. Extensive checks performed in our previous paper 15] demonstrated that our ASA-LMTO based approach yields results for the second order response functions of comparable accuracy as the full-potential linear augmented plane-wave method (FLAPW) method [20,21]. The formulas used here to calculate $\chi^{(2)}$ were given in a previous publication. [15] They are based on the so-called "length- 
gauge" formalism of Sipe and Ghahramani, [13] and Aversa and Sipe [14]. This formalism uses the independent particle approximation and is presently restricted to undoped semiconductors. Also, it does not include the local field effects. This method has several apparent advantages, e.g., the manifest absence of unphysical singularities of nonlinear optical responses in the zero-frequency limit and the explicit satisfaction of the Kleinman relations. [22]

The self-consistent LMTO electronic structure calculations were carried out within the framework of the local density approximation (LDA) in the density functional theory. The exchange-correlation potential has been taken in the form of Hedin and Lundqvist. [23] One of the central problems in calculation of optical response functions in semiconductors is the problem of the gap corrections in the LDA 15,20,21,24,25] which appears from the fact that response functions have to deal with the actual quasiparticle excitations rather than the Kohn-Sham eigenstates. The presently most accurate approach, the GW method, is unfortunately rather computationally demanding because it needs calculations of matrix elements of the nonlocal self-energy operator in terms of the one particle Green's function and the screened Coulomb interaction which requires itself calculation of the inverse dielectric response function including local field effects. [26] In practice, a simplified approach, the so-called "scissors operator" is often used. It assumes the rigid shift in energy $\Delta$ of the conduction band with subsequent renormalization of the velocity (momentum) operator matrix elements (see, e.g., Refs. [15,20,24] for details). In our previous paper [15] we noted that this approach is not entirely satisfactory because it breaks the consistency between the eigenvectors and eigenvalues of the one particle Hamiltonian. This manifests itself rather clearly when applying this approach to the calculation of effective masses. In a rigid shift, the masses should not change, but applying the "scissors" renormalization to the momentum matrix elements and using the well-known $\mathbf{k} \cdot \mathbf{p}$ expression for the inverse masses we see that the effective mass decreases.

We proposed an alternative approach, which consists in adding semi-empirical corrections to the diagonal elements of the LMTO. In some sense, it is an attempt of describing the scissor's operator $\sum_{c k} \Delta_{c k}|c k\rangle\langle c k|$, in which $|c k\rangle$ are the conduction band states and $\Delta_{c k}$ their shifts, explicitly at the level of the Hamiltonian. The approach is based on the observation that the states at the bottom of the conduction band in tetrahedrally bonded semiconductors have a characteristic predominance of certain LMTO basis orbitals in their wave functions: e.g., cation $s$ - at $\Gamma$ and a mixture of cation $s$ - and empty sphere $s$ - orbitals at the $X$ - point of the zincblende BZ. Therefore, by shifting these $s$ orbitals, one shifts the corresponding states. The states at $L$ typically behave intermediately between those at $\Gamma$ and $X$. Thus, an advantage is that one can include k- dependent shifts if these are known. This alternative approach is closely related to the one suggested by Christensen [27] in which it is further used that only $s$ - states are sensitive to sharply localized $\delta$-function-like potentials. In our previous paper 15 we showed that better results for the frequency dependent SHG (at least for GaAs and GaP) can be obtained in this way. However, this procedure allows us only to shift the lowest conduction bands while for some materials the shift of the whole set of conduction bands is a better approximation (e.g., for $\mathrm{SiC}$, see Ref. [16] for details). In other words, it is a reasonable approach only if one is interested in the highest valence band and the lowest conduction band exclusively. Its success for GaAs and GaP observed in our previous work is thus based on the fact that these bands tend to dominate the second order response functions in that material but this is not a general fact for all semiconductors.

In the present study, in order to study trends, it is important to follow a systematic and consistent treatment for all the compounds, including those for which detailed band gap information at different $\mathbf{k}$ - points is not available. Therefore, the bulk of our results was obtained using the simple "scissors operator" approach. Unfortunately, we cannot use that approach directly for the case of $\mathrm{CdGeAs}_{2}$ (where LDA does not give any gap at all). As a result, the bands in $\mathrm{CdGeAs}_{2}$ have a completely changed topology near the "overlap" of the valence and conduction bands at $\Gamma$. To deal with this case we first applied a shift of the Hamiltonian $s$ - states to get some "bare minimum" gap of at least $0.3 \mathrm{eV}$ and subsequently applied the usual scissors operator to obtain the experimental gap values.

We emphasize that the primary purpose of this paper is to study the chemical and structural effects on the electronic structure and $\chi^{(2)}$ functions. The above described problems with the gap corrections, although not handled to our entire satisfaction, are expected to only have a minor effect on the main conclusions of our work as long as a consistent treatment is followed.

The detailed description of the procedure for calculating the linear response and frequency dependent SHG has been described elsewhere (see, e.g., Ref. [15]). The real part of the frequency dependent response functions has been obtained from the imaginary part by using the the Kramers-Kronig transformation. For the zero-frequency limit of SHG we use the simplified and computationally more convenient formulas. It avoids the need for calculation of the response function in a wide frequency region required for an accurate application of the Kramers-Kronig transformation and is less singular as regards k- point summations, so it can be converged with fewer terms. The k- space integration may be limited to the irreducible wedge of the Brillouin zone by performing a prior symmetrization of the product of the three momentum matrix elements over all the transformation of the point group of the crystal.

For the frequency dependent SHG, we use the usual tetrahedron scheme of the integration with linear interpolation of the band energies and the products of the matrix elements. For the zero-frequency limit on the other hand, we employ a semi-analytical linear interpolation scheme which is more efficient and produces a smaller error. 15. 


\section{RESULTS}

\section{A. Crystal structure and electronic bands}

The chalcopyrite $\mathrm{ABC}_{2}$ materials (with chemical composition II-IV-V 2 and I-III-VI $\mathrm{VI}_{2}$ can be obtained from III-V compound and II-VI compounds respectively by replacing every two group III (II) atoms per cell by a II and IV (I and III) atom. For example, from GaP we derive $\mathrm{ZnGeP}_{2}$ by this chemical substitution. Their crystal structure is a body-centered tetragonal lattice which has eight atoms per unit cell. It can be thought of as a tetragonally distorted $\mathrm{A}_{2} \mathrm{~B}_{2}$ ( or $2+2$ ) face centered cubic (fcc) superlattice in the $\{201\}$ direction of the cations $\mathrm{A}$ and $\mathrm{B}$ with an interpenetrating fcc lattice of common anions $\mathrm{C}$ displaced by $(1 / 4,1 / 4, u)$. The structural parameters are the lattice constant $a$ which corresponds to the cubic lattice constant of the zincblende structure from which the chalcopyrite structure is derived, the ratio $\eta=c / a$, and the internal displacement parameter $u$. In the ideal structure $\eta=2$ and $u=1 / 4$. The nonideal value of $u$ is due to the distortion of the anion sublattice involving a shift by each anion away from one neighboring cation in the direction of the another cation (of different sort). It was shown that such a shift of anions is a consequence of atomic sizes [28 30]. Usually the column-II atom is larger than the column-IV one, and the anions are closer to $\mathrm{B}$ than to $\mathrm{A}$ atoms.

In principle, one can obtain the $a, \eta$ and $u$ parameters by total energy minimization. It was shown in a previous paper by one of the authors that such a relaxation can significantly affect the band structure 31. We did not do such a relaxation in the present work but preferred to use the available experimental values of these structural parameters. Some comparisons to the ideal structure were also carried out. Table if shows experimental values of the lattice parameters $a, \eta$, and $u$ for several chalcopyrites $\mathrm{ABC}_{2}$ from the considered group. The most complete set of parameters can be found in Refs. [32] and [33]. The early measurements of Ref. [32] did not pay too much attention on detailed measurements of the parameter $u$ but the measured values of the parameters $a$ and $\eta$ are very close in both the references. Below we show that the distortions from the ideal chalcopyrite structure $(\eta=2, u=1 / 4)$ can be neglected only when they are small. Otherwise, they can change significantly the value of the gaps and the SHG. It would be interesting to mention that the substitution of phosphorus by arsenicum increases the lattice constant $a$ by the factor of 3.5-4\% while the value of $\eta=c / a$ changes by less than $1 \%$. The substitution of Ge by Si does not change either $a$ or $\eta$ by more than $1.5 \%$.

Table III shows the experimental values of the minimum band gaps in these materials from Ref. [33] compared to our LDA gaps and their difference in the first three columns. The nature of the band gaps varies from direct to pseudodirect or indirect. By pseudodirect is meant that the gap is nominally direct but corresponds to a weak, almost forbidden, optical transition. In the chalcopyrites, this situation can occur because the zone edge states of the parent III-V zincblende compound at $X^{z b}$ are folded onto the $\Gamma$-point in the chalcopyrite structure. Thus, when the zincblende $X_{1 c}$ state is lower than the direct gap at $\Gamma$ (which happens in GaP), a pseudodirect gap is expected in a chalcopyrite corresponding to a small perturbation from GaP. The correspondence between states in chalcopyrite notation and the states in zincblende is as follows: the conduction band minimum at $X, X_{1 c}^{z b} \mapsto \Gamma_{3}$, the next higher state at $X, X_{3 c}^{z b} \mapsto \Gamma_{2}$ and $\Gamma_{1 c}^{z b} \mapsto \Gamma_{1}$. The latter is predominantly s-like while the other two have important pcontributions. The optical matrix elements between the crystal-field splitted valence band maxima $\Gamma_{4}$ or $\Gamma_{5}$, which are both p-like, and the $\Gamma_{1}$ conduction band state are large while the ones with $\Gamma_{3}$ and $\Gamma_{2}$ are small. By inspection of the band dispersion, or the matrix elements, or the eigenvectors, one can easily identify the nature of the lowest conduction band at $\Gamma$ as being $\Gamma_{1}$ or $\Gamma_{3}$ like. Our calculated gaps indicted as being pseudodirect in fact correspond to the $\Gamma_{3}$ minimum while the direct ones correspond to a $\Gamma_{1}$ minimum. The experimental assignments are mainly based on the optical absorption being weak or strong [34 along with some general theoretical considerations based on zone folding arguments of the type explained above. Experimentally, it is very difficult to distinguish between a pseudodirect and a truly indirect gap.

Although all the LDA gaps are lower than the experiment by about $1 \mathrm{eV}$, we note that the assignments of the direct or pseudodirect nature agree with experiment with two exceptions. We find $\mathrm{ZnGeP}_{2}$ to be actually indirect with a conduction band minimum location at a point near to the folded $X$-point. This will be further discussed elsewhere [35] and is still in agreement with the absorption measurements indicating a weak transition. Also, we find ZnSiAs 2 to be direct while experiment indicates a pseudodirect gap. We note however, that in this case, the $\Gamma_{3}$ pseudodirect gap as well as the lowest indirect gap in our calculation are only $0.1 \mathrm{eV}$ above the direct $\Gamma_{1}$ gap. Errors of the order of $0.1 \mathrm{eV}$ in the ordering of the conduction band states are common, a well-known example being Ge, for which it was found that subtle effects such as core-polarization [36] need to be included to obtain the correct ordering.

We note that if we order these semiconductors according to increasing gap, the lower gap ones are direct, the higher gap ones are pseudodirect (or indirect) with the crossing taking place at about $1.7 \mathrm{eV}$, i.e., $\mathrm{CdGeP}_{2}$ and $\mathrm{ZnSiAs}_{2}$ being borderline cases. From the above discussion relating the bands to those of the parent III-V compounds, the pseudodirect situation is clearly expected in $\mathrm{ZnSiP}_{2}$, because $\mathrm{Zn}$ is in the same row of the periodic table as Ga and 
the Si s-state occur higher than the Si-p states $\left(\Gamma_{2^{\prime}}>\Gamma_{15}\right)$. Thus one expects the $\Gamma_{1}$ state to be mainly Zn- like, and a a small perturbation from $\mathrm{GaP}$ is expected. In $\mathrm{ZnGeAs}_{2}$, on the other hand, we clearly expect a truly direct ( $\Gamma_{1}$-like) gap because GaAs is direct. Next, we note that $\mathrm{Cd}$ being a heavier element has lower s-like states than $\mathrm{Zn}$ and thus replacement of $\mathrm{Zn}$ by $\mathrm{Cd}$ will tend to make the gap direct as opposed to pseudodirect. Ge also has a lower $\Gamma_{1}$ state than Si. Thus replacing Si by Ge will also tend to make the gap direct. This is consistent with the borderline case $\mathrm{CdGeP}_{2}$ being direct. Also, in $\mathrm{ZnGeP}_{2}$, we find the $\Gamma_{1}$ state slightly below the $\Gamma_{3}$ but nevertheless it is indirect with a minimum gap at another k- point. Again, in this case the difference between direct and indirect or pseudodirect gaps is of the order of $0.1 \mathrm{eV}$ only. In conclusion, the nature of the gaps is generally well described by our LDA calculations.

Next, we consider the gap correction from the LDA. We note that both the $\mathrm{Zn}$ and $\mathrm{Cd}$ compounds have a gap correction $\Delta$ of about $1 \mathrm{eV}\left(\Delta\right.$ varies from $0.83 \mathrm{eV}$ for $\mathrm{ZnSiAs}_{2}$ to $1.13 \mathrm{eV}$ for $\left.\mathrm{CdSiAs}_{2}\right)$. This is consistent with the gap corrections of GaP $(1.2 \mathrm{eV})$, [37] GaAs [37,38] (0.9 eV), and Zn-VI compounds [39] (1.2-1.6 eV).

Other aspects of our band structures not discussed here are in general agreement with those of previous studies, [28 30,40] for some of these compounds although there are some differences in the location of the Zn3d band. Thus, apart from the gap corrections, which for simplicity we take from experiment and apply as a "scissors" shift to all conduction states, our band structures seem to describe the electronic structure of these systems rather well. In any rate, the optical response functions considered in this paper are not very sensitive to the fine band structure details such as changes from direct to indirect at the level of $0.1 \mathrm{eV}$, because they are derived from integrals over the whole Brillouin zone. Also, the contributions of the $\mathrm{Zn}$ or $\mathrm{Cd}$ d-bands in the optical transitions determining $\chi^{(2)}$ are quite negligible being far too deep although they do have an indirect effect on the lattice constants and the states near the valence band maximum via their hybridisation with the d-bands.

\section{B. Linear optical response and birefringence}

For practical applications in SHG and OPO's an important quantity is the birefringence because it enters in the phase-matching condition. It can be calculated from the linear response functions from which the anisotropy of the index of refraction is obtained. Figure 1 shows the imaginary part of the dielectric function (DF) $\varepsilon_{2}(\omega)$ for $\mathrm{ZnGeP}_{2}$ and $\mathrm{CdGeAs}_{2}$ in a wide energy region and for different light polarizations. In this subsection we concentrate exclusively on these two materials because of recent experimental measurements of high accuracy of frequency and temperature dependence of birefringence. [2, 3.,7] In general, the $\varepsilon_{2}(\omega)$ 's are rather similar in these materials, except for the difference in the gap value. It consists mostly of one broad hump with a few additional fine structures which depend on the light polarization.

The birefringence is the difference between the extraordinary and ordinary refraction indices, $\Delta n=n_{e}-n_{o}$, where $n_{e}$ is the index of refraction for an electric field oriented along the c-axis and $n_{o}$ is the index of refraction for an electric field perpendicular to the c-axis. It is positive for both the materials $\mathrm{ZnGeP}_{2}$ and $\mathrm{CdGeAs}_{2}$. In the low energy region $(\sim 0.1 \mathrm{eV})$ phonon absorption is starting to play an important role, and this was clearly observed in the temperature dependence of the birefringence in Refs. [2, 3, :7]. We cannot study the temperature dependence in the present publication. However, when the temperature is changing between $14 \mathrm{~K}$ and $450 \mathrm{~K}$, the change of the birefringence at a given frequency is not larger than 10-15\% of its value at room temperature, which is about the error of our present estimation which is limited in its accuracy among others by the uncertainties in the gap corrections and by the neglect of local field effects.

Figure 2 shows $\Delta n$ in a wide energy region. Of course, in practice the birefringence is important only in the non-absorbing region, i.e., below the gap. The presense of an absorption makes the use of the nonlinear crystal in OPO's or frequency doubling device quite difficult or impossible. However, such a curve is illustrative to show the general aspects of the difference of the DF for different polarization. One may note that the general shape of the curves for $\mathrm{ZnGeP}_{2}$ and $\mathrm{CdGeAs}_{2}$ is rather similar, indicating the same frequency regions where the $\epsilon_{2}(\omega)$ functions are enhanced or decreased in one polarization or the other. This, of course, is due to the similarities in their underlying band structures at the $\mathrm{eV}$ scale. Nevertheless, we can recognize a stronger negative peak around $2.5-3.0 \mathrm{eV}$ in $\mathrm{CdGeAs}_{2}$ than in $\mathrm{ZnGeP}_{2}$ and a higher positive value in the low energy region.

The birefringence of $\mathrm{ZnGeP}_{2}$ and $\mathrm{CdGeAs}_{2}$ in the non-absorbing energy region together with experimental data is shown in Figures 3 and 4 . As one could expect the calculated curve is growing quadratically with energy (in the experiment $\Delta n$ starts to grow again at low energies due to the phonon absorption). The curve is continuing to grow with energy below the gap reaching a non-monotonic behavior when the interband absorption processes start. For both $\mathrm{ZnGeP}_{2}$ and $\mathrm{CdGeAs}_{2}$ we predict fairly accurate values of the birefringence to within about $10 \%$ in the middle of the gap region. It correctly accounts for the fact that it is about twice larger in $\mathrm{CdGeAs}_{2}$ than in $\mathrm{ZnGeP}_{2}$. For $\mathrm{ZnGeP}_{2}$ the numerical values are in quite reasonable agreement with experiment in all the non-absorbing region. For 
$\mathrm{CdGeAs}_{2}$ the agreement with measurements is good in a low energy region $(0.1-0.3 \mathrm{eV})$ but at higher energies the experimental curve starts to grow faster than the theoretical one. The reason for this disagreement can be different, e.g., (i) the measurements could be prepared on a crystal which is not perfect and contains impurities and cracks which increase the scattering; (ii) the excitons (non included in our calculations) could be important in this materials and give a significant contribution in refraction index near the band gap edge; (iii) our calculations of the dielectric function for $\mathrm{CdGeAs}_{2}$ might be not accurate enough. Therefore, our calculations of birefringence should be considered as qualitative estimates only. Nevertheless, it is gratifying to see that even such an estimation exhibits the correct trends for the dependence of the birefringence on the chemical composition in these two chalcopyrites. This shows that our calculations will be predictive for other chalcopyrites and may be used for preliminary investigations of the phase-matching conditions in these and related materials.

\section{Static limit of SHG}

In this section we focus on the results for the SHG coefficients in the zero frequency limit. For the material with the point group $\overline{4} 2 m$ there are only two independent component of the SHG tensor, namely, the 123 and 312 components $(1,2$, and 3 refer to the $\mathrm{x}, \mathrm{y}$, and $\mathrm{z}$ axes respectively, which are chosen along the cubic axes). In the static limit, these two components are becoming equal according to Kleinman "permutation" symmetry, 22 which dictates additional relations between tensorial components beyond the purely crystallographic symmetry. For a non-ideal chalcopyrite structure (when $u \neq 1 / 4$ ) the point group symmetry is distorted and some additional components of SHG (e.g., 311 or 131) appear. However, direct numerical calculations show that these additional components are not larger than 3-5 $\%$ of the main 123 component for the values of $u$ corresponding to real chalcopyrites. Therefore, we ignore them in the bulk of our results and make all the calculations for the $\overline{4} 2 \mathrm{~m}$ point group. For the considered ternary chalcopyrite semiconductors including the orbitals with angular moments $l_{\max }=3$ in the basis set changes the results by the factor of $5-10 \%$ only. In most of our calculations we neglect them including orbitals with moments up to $l_{\max }=2$.

In addition to the gaps discussed earlier, Table 1 shows the values of the calculated and measured (where available) SHG in the zero frequency limit in $\mathrm{ABC}_{2}$ compounds $(\mathrm{A}=\mathrm{Zn}, \mathrm{Cd} ; \mathrm{B}=\mathrm{Ge}, \mathrm{Si} ; \mathrm{C}=\mathrm{As}, \mathrm{P})$. We recall that all values were calculated using the "scissor" approach with a rigid shift of the conduction bands chosen such that the minimum gap is adjusted to the experimental value.

First, we discuss the trends with chemical element. One can see that substitution of $\mathrm{P}$ by As keeping the other elements fixed increases $\chi^{(2)}$ in all cases. This is also consistent with the values of GaAs (105 pm/V) and GaP (48 $\mathrm{pm} / \mathrm{V}$ ) calculated using the same approach (LDA+scissors) in Ref. [15]. Similarly, substitution of Si by Ge and Zn by $\mathrm{Cd}$ increases $\chi^{(2)}$. Second, one may notice that this is correlated with the band gaps. Substitution of As by $\mathrm{P}$, or Ge by $\mathrm{Si}$, or $\mathrm{Cd}$ by $\mathrm{Zn}$, each increase the gap and decrease $\chi^{(2)}$. However, these qualitative trends cannot easily be turned into quantitative scaling laws. If one more carefully considers the ratios of the gaps and the ratios of $\chi^{(2)}$ for each of the above substitutions, no inverse power law between gaps and $\chi^{(2)}$ can be extracted in the remaining four element combinations. For example, for substitution of As to $\mathrm{P}$, the ratios of the gaps for $\mathrm{ZnGe}, \mathrm{ZnSi}, \mathrm{CdGe}$ and $\mathrm{CdSi}$ are 1.8, 1.2, 3.0 and 1.4, respectively, but the inverse ratio of the $\chi^{(2)}$ 's are 1.8, 1.7, 4.0 and 1.9. On the basis of the theoretical expressions, [15] one might have guessed a scaling with the third power of the gap because three energy denominators occur. Clearly, such a strong scaling with the gap is not present. The reason for this is that there are several terms with positive and negative contributions which to a great extent cancel each other and secondly, the $\chi^{(2)}$ is not dominated by the terms involving the minimum gap but arises from a $\mathbf{k}$ - point summation over the whole Brillouin zone and a sum over several band-to-band terms. Thus, no simple scaling with the gaps emerge.

The smallest value of the gap occurs for $\mathrm{CdGeAs}_{2}$ which also has the largest $\chi^{(2)}$. From the above discussion of the trends, one can certainly say that $\mathrm{CdGeAs}_{2}$ has the mostly favorable chemical combination of the elements among the class of chalcopyrites considered here. However, these arguments do not suffice to explain why the second harmonic generation coefficient in $\mathrm{CdGeAs}_{2}$ is so exceptionally large.

Before doing a more detailed analysis of the frequency dependent $\chi^{(2)}$ in the next section we first analyze the next two columns of the table where we put separately interband versus intraband contributions in SHG. The meaning of these terms needs perhaps some clarification. In general, the formulas for second order response include many terms, and its division in several groups called intra- or interband is rather conventional and depends somewhat on the formulation of the problem. For instance, it appears at first to be different in the formulas using the length-gauge and the momentum gauge formulations. The most convincing decomposition based on a careful analysis of the underlying time dependent perturbation theory is the following [13 15]: the "interband" contribution corresponds to polarization loop where all the three Green's function belong to different electronic bands, while the "intraband" processes include the modulation of the linear response by the intraband motion as well as the modification of the intraband motion by the interband polarization processes. 
Analysis of the intraband and interband $\chi^{(2)}$ contributions shows that, (i) for all the considered $\mathrm{ABC}_{2}$ compounds the interband contribution is negative while intraband is positive, therefore, these two terms work in opposite directions; (ii) for all the materials except $\mathrm{CdGeAs}_{2}$ and $\mathrm{CdSiAs}_{2}$ both absolute values of the intraband and interband terms are larger than the resulting total SHG; (iii) the absolute value of the intraband term is always larger than those of interband, i.e., the resulting total SHG is positive. We also notice that $\mathrm{CdGeAs}_{2}$ and $\mathrm{CdSiAs}_{2}$ are exceptions which have an exceptionally small value of $\chi_{\text {inter }}^{(2)}$, i.e., the intraband term is dominating the SHG in the zero frequency limit. This term for $\mathrm{CdGeAs}_{2}$ is nearly the same as in $\mathrm{ZnGeAs}_{2}$. However, there is no interband contribution to compensate it. This explains why the second response function is so large in this material. The value of $\chi_{\text {intra }}^{(2)}$ in $\mathrm{CdSiAs}_{2}$ is a few times smaller than in $\mathrm{CdGeAs}_{2}$ because of differences in the value of the gap.

Another point of interest is a comparison with the parent III-V compounds GaAs and GaP. The values obtained at the same level of calculation [15] have already been given above. Clearly, all phosphides considered here have a higher value of $\chi^{(2)}$ than GaP. They also have consistently lower gaps. For the arsenides, we also find the $\chi^{(2)}$ values to be consistently higher or equal in the chalcopyrites than in GaAs, and to have lower gaps, except for $\mathrm{ZnSiAs}_{2}$ which has a slightly higher gap and the same value of $\chi^{(2)}$ as GaAs. The chalcopyrite type chemical substitution III-V to II-IV-V $V_{2}$ thus clearly appears to be favorable for $\chi^{(2)}$. In addition, and even more important in practice, the anisotropy of the chalcopyrite crystal structure allows for angular phase-matching while this is not possible in the cubic zincblende crystals.

The comparison of calculations with experiment is somewhat complicated because the reported experimental results for these materials differ considerably among each other. The last two columns of Table II show experimental values of SHG from the two different handbooks (Ref. [11] and Ref. [10]). The values from Ref. [10] are systematically higher than those from Ref. [11] by a factor of 1.3-1.4. The reason of such a discrepancy is not completely clear. One possible explanation is that most of the original values of SHG were measured relatively to quartz, and the absolute value of second harmonic generation of pure quartz has been remeasured a few times (see Ref. 41] for more recently recommended value of SHG in quartz). For $\mathrm{ZnGeP}_{2}, \mathrm{ZnSiAs}_{2}$, and $\mathrm{CdGeP}_{2}$ our calculations are closer to Ref. [11] while for $\mathrm{CdGeAs}_{2}$ they agree well with Ref. 110]. In general, we know that the "scissors" corrected values are overcorrected, i.e., the actual value of SHG has to be higher [15,16]. Such an overcorrection is an intrinsic problem of the "scissors" approach. With these cautions in mind, some of our values for the cases where no experimental values are available can be used as predictions.

\section{Frequency dependent SHG}

In order to better understand the origin of the relative magnitudes of the intra- and interband contributions, we now consider the frequency dependent $\chi^{(2)}$ functions, or, more precisely, their imaginary part from which the real part and in particular its static value can be obtained by a Kramers-Kronig transformation. Figures 5 and 6 show the imaginary part of the frequency dependent $\chi^{(2)}(\omega)$ for the 123 and 312 components in $\mathrm{ZnGeP}_{2}$ and $\mathrm{CdGeAs}_{2}$. First, it would be worth to mention that the SHG curves for 123 and 312 components look very similar being different just in fine structure details. This is not very surprising because in the initial zincblende material which was used to derive the chalcopyrite material by chemical substitutions these two components were equal at arbitrary frequency. The shape of the curves is also qualitatively very similar in the two materials. It consists of a broad hump with positive SHG values between the half of the band gap and 1.5-2 eV, then it changes sign and exhibits another broad dip between 2 and $3 \mathrm{eV}$. At energies higher than $4 \mathrm{eV}$ the imaginary part of SHG drops to zero very fast. The amplitudes of both broad features are somewhat higher in $\mathrm{CdGeAs}_{2}$ than in $\mathrm{ZnGeP}_{2}$. This is probably due to the smaller value of the gap in the first material. $\operatorname{Im} \chi^{(2)}(\omega)$ for the other ternary pnictides $\mathrm{ABC}_{2}$ considered above are looking very similar, and we do not show them.

As we see, the analysis of $\operatorname{Im} \chi^{(2)}(\omega)$ does not answer the question concerning the origin of the extremely large value of the zero frequency SHG in $\mathrm{CdGeAs}_{2}$. To this end, it is more useful to analyze different contributions to the total SHG curves. Figures 7 and 8 show the intraband and interband contribution separately. For $\mathrm{ZnGeP}_{2}$ both the interband and intraband contributions in the most interesting energy region below $4 \mathrm{eV}$ (for the Kramers-Kronig integral which gives the zero frequency value) do not change the sign (the first is negative, the other is positive). This means that both the inter- and intraband contributions to the static SHG are large and have opposite signs. In $\mathrm{CdGeAs}_{2}$ the situation is different. While the intraband term behaves in the similar way to those in $\mathrm{ZnGeP}_{2}$ (does not change the sign below $4 \mathrm{eV}$ ), the interband term is positive between 0.3 and $1.1 \mathrm{eV}$ and negative between 1.1 and $3 \mathrm{eV}$. Thus, the low energy region (below $4 \mathrm{eV}$ ) of the interband term by itself already exhibits a good deal of compensation between positive and negative values when the integral appearing in the Kramers-Kronig transformation is calculated. There is no simple qualitative explanation of the fact why the resulting Kramers-Kronig integral which gives the zero frequency value of the interband contribution to SHG is as small as the numerical calculations show: 
less than $0.5 \%$ of the intraband term in our calculations, see the previous section). This appears to be a rather coincidental interplay between different interband contributions. The important fact is that the contributions from different groups of interband transitions in this material have different sign, and they compensate each other in the Kramers-Kronig integral.

This example shows that a small value of the gap may be favorable for but is clearly not sufficient or the dominant contributing factor to obtain a large value of SHG. The value of the SHG results from a delicate balance between different contributions too complicated to capture in a simple scaling law with the gap. Unfortunately, we cannot presently further reduce this question to a simple explanation in terms of particular band-to-band transitions. Nevertheless, it shows that the entire band structure matters, not simply the gaps. Therefore, there appears, at least in principle, to be an ample room for optimization of $\chi^{(2)}$ by band structure engineering. Of course, we cannot control the signs of different interband contributions in a definite bulk chemical compound at will even with quite elaborate chemical substitutions. However, further opportunities to modify the band structures could exist for semiconducting heterostructures, such as multiple quantum wells, superlattices, layered materials, quantum dots, etc. In these structures the energies of the transitions between different levels and their matrix elements can to some extent be controlled by geometrical parameters of the structure. For example, it has already been shown that the asymmetric quantum wells can display very large values of second order optical response (see, e.g., Refs. 42, 43,). However, the situation in these systems is complicated, and theoretical studies show that excitonic effects play an important role, 44 46 in addition to the single electron part considered in our work. Nevertheless, the idea of parametrically controlled heterostructures with large $\chi^{(2)}$ is a challenging task which we hope to pursue in future work because of the practical importance of obtaining materials with higher $\chi^{(2)}$ values.

\section{E. Structure dependence}

As we already mentioned above, the distortions from the ideal chalcopyrite structure can be neglected in calculations of optical spectra and SHG only if they are small enough as in the case of $\mathrm{ZnGeP}_{2}$. Figure 9 compares the electronic bands for $\mathrm{ZnGeP}_{2}$ along the $Z-\Gamma$ and $\Gamma-X$ lines calculated in two different crystal structures, namely, the real experimental structure (with the parameters from Table II), and the ideal chalcopyrite structure with the same lattice constant $a$. The band structures look very similar being different only in some fine details (e.g., the splitting of the upper valence band at the $\Gamma$ - point is different in the two cases due to different crystal fields). Both crystals have indirect $(\Gamma-X)$ band gaps of nearly the same value. The zero-frequency SHG values calculated in LDA are also very close $(102 \mathrm{pm} / \mathrm{V}$ for the experimental structure and $106 \mathrm{pm} / \mathrm{V}$ for ideal chalcopyrite, i.e., the difference between them is only about $4 \%$ ).

In materials with larger distortions the situation is different. One has to study how the deviations of both the parameters $\eta$ and $u$ from those of the ideal chalcopyrite structure $(\eta=2, u=1 / 4)$ affect both the band structure and optical constants. Figure 10 shows how the value of the parameter $u$ affects the electronic bands in $\mathrm{CdSiP}_{2}($ this compound is the most illustrative because the deviations of both $\eta$ and $u$ are the largest ones when compared with other materials of the considered group). Except for some fine details, the effect of $u$ can be considered as a rigid shift upwards (by about $0.4 \mathrm{eV}$ ) of the low conduction bands. The character of the gap (pseudodirect) does not change with $u$. The behavior of the band structure is completely different when we change the parameter $\eta$ (Fig. 11). Namely, when the value of $\eta$ is approaching 2, the two lowest conduction bands are changing their positions, and the band gap is becoming direct as opposed to indirect. This illustrates the differences of the behavior of the electronic spectra with changes in $\eta$ and $u$.

For the other materials the situation is quite similar. The change of $u$ produces nearly rigid shift of the few lowest conduction bands while the changes of the parameter $\eta$ can mix these bands and change the character of the band gap. Moreover, the value of the rigid shift is nearly linear in $(u-1 / 4)$ and depends very weakly on the material. Thie means that the deformation potential associated with this structural change is almost constant in this class of compounds. Why this is so is presently not clear although it is not entirely unexpected since, in general, these materials are of course all closely related to GaAs or GaP.

In order to study how these changes affect the optical constants we calculated the zero frequency SHG coefficients at different values of $\eta$ and $u$. For the band structures of $\mathrm{CdSiP}_{2}$ shown in Figures 10 and 11 this calculation gives $\chi^{(2)}=73 \mathrm{pm} / \mathrm{V}$ for the real experimental structure, $\chi^{(2)}=90 \mathrm{pm} / \mathrm{V}$ for $\eta=1.836$ and $u=1 / 4$, and $\chi^{(2)}=113$ $\mathrm{pm} / \mathrm{V}$ for the ideal chalcopyrite structure. The value of the lattice constant $a$ and the value of the gap correction used was the same in all the three cases. These calculations illustrate qualitatively how SHG depends on distortions of the ideal chalcopyrite structure. In the considered case both the deviations of $\eta$ and $u$ tend to decrease the value of SHG.

Another interesting question is to study whether or not the chalcopyrite type of ordering of the cations plays a 
special role in high $\chi^{(2)}$ values in these materials. For this purpose we considered an alternative crystal structure, a (001) oriented $1+1$ superlattice $\mathrm{AC}+\mathrm{BC}$, which still maintains the local tetrahedral environment of the chalcopyrite where each anion is surrounded by two cations of each type but which nevertheless exhibits a different ordering vector. This superlattice structure has the same overall chemical formula and stoichiometry as the chalcopyrite structure.

Figure 12 shows the LDA band structures of the $1+1 \mathrm{ZnGeP}_{2}$ superlattice along the same directions of the Brillouin zone as in Fig. 9. The real cell for the superlattice is a tetrahedral one which is twice smaller than the cell for the chalcopyrite structure. We also calculated the electronic structure of $1+1$ superlattices for other $\mathrm{ABC}_{2}$ materials considered before. However, in some materials which exhibit a small LDA gap in the chalcopyrite structure, the gap for the $1+1$ superlattice becomes negative. Here we show the results for $\mathrm{ZnGeP}_{2}$ only in order to illustrate how the band structure changes with modifications in the crystal structure. The gap is much smaller in the $1+1$ structure $\left(E_{g}^{L D A}=0.3 \mathrm{eV}\right)$ than in the chalcopyrite $\left(E_{g}^{L D A}=1.16 \mathrm{eV}\right)$. As a result, the zero frequency SHG is about $190 \mathrm{pm} / \mathrm{V}$ for the superlattice, i.e., about twice higher than SHG in the chalcopyrite $\mathrm{ZnGeP}_{2}$ (we used the same value of the gap correction $(\Delta=0.89 \mathrm{eV})$ for the superlattice structure which has been used before for the chalcopyrite $\mathrm{ZnGeP}_{2}$ ).

While this is at first sight promising for obtaining higher $\chi^{(2)}$ we caution that this structure is unlikely to be stable. The atomic sizes of cations are different, and one can expect an occurance of alternatingly compressive and tensile biaxial strain in the cation layers. The calculations show that the difference of the total energy per chemical unit between the superlattice and the chalcopyrite structure is $\Delta E_{t o t}=0.204 \mathrm{eV}$, i.e., is rather large. This confirms our hypothesis that the alternative structure is not stable with respect to different structural rearrangements minimizing the total energy.

\section{CONCLUSIONS}

The linear and nonlinear optical properties for important group of chalcopyrites $\mathrm{ABC}_{2}(\mathrm{~A}=\mathrm{Zn}, \mathrm{Cd} ; \mathrm{B}=\mathrm{Ge}, \mathrm{Si}$; $\mathrm{C}=\mathrm{As}, \mathrm{P}$ ) have been calculated over a wide energy range. We studied any possible combination of $\mathrm{A}, \mathrm{B}$, and $\mathrm{C}$. This allowed us to study the trends in the second order optical response with chemical composition. The results for the zerofrequency limit of SHG are in good agreement with available experimental results. The calculated birefringence for $\mathrm{ZnGeP}_{2}$ and $\mathrm{CdGeAs}_{2}$ also shows a fair agreement with recent experimental data in the energy region corresponding to the middle of the gap. For all the considered compounds the second harmonic generation coefficient $\chi^{(2)}$ is of the order of 100-200 pm/V, i.e., of the order of and in fact larger than the SHG in the initial zincblende material (GaAs or $\mathrm{GaP}$ ) from which these compounds were created by chemical substitutions. The only exception is $\mathrm{CdGeAs}_{2}$, which has much higher nonlinear response $(\sim 500 \mathrm{pm} / \mathrm{V})$ than all the other materials from this group. This value of the SHG cannot be explained only by a small value of the band gap. It appears as a result of a very delicate balance between different terms which contribute to second order response.

The results of the calculations are rather stable with respect to small structural modifications. They do not change much with distortions of the ideal chalcopyrite structure in materials in which $\eta$ and $u$ are close to those in ideal chalcopyrite structure. However, when these deviations are large (like in $\mathrm{CdSiP}_{2}$ ) the gaps as well as SHG's can be strongly affected by the values of $\eta$ and $u$ chosen in calculations, and the ideal structure does not describe a real experimental situation correctly. The LDA calculations for the (001)- oriented $1+1$ superlattice which has the same overall chemical formula and stoichiometry as the chalcopyrite structure exhibits a much smaller LDA band gap than the chalcopyrite structure and larger values of the SHG coefficients. However, this structure has a very high total energy which is probably related to large strains in the cation layers. These strains should make the structure unstable with respect to different structural rearrangements.

In conclusion, we can certainly say that a small value of the gap is favorable for larger SHG. However, this is by no means sufficient for quantitative predictions of the $\chi^{(2)}$ values from the known values of the gaps. Within this family of compounds all calculated in essentially the same approximation, no clear scaling of calculated $\chi^{(2)}$ values with a power law of the gap was obtained. Because of the experimental uncertainties in determining $\chi^{(2)}$ values for compounds with different degree of crystalline ideality and different optimization of phase-matching conditions, we consider this test as a more reliable test of such scaling laws than those based on experimental values. The origin of $\chi^{(2)}$ in terms of the underlying band structure is clearly too complex for such a simple minded extrapolation to be valid. One has to take into account all the contributing terms and analyze them carefully. Even then, gaining understanding is far from easy and we were only partially successful at unraveling the origin of the high value of $\chi^{(2)}$ for $\mathrm{CdGeAs}_{2}$. Nevertheless, we hope that our present analysis in terms of the intra- and interband terms provides at least a first step towards such understanding. Both the intra- and interband terms are generally found to be decreasing with increases of the gap because both term contain some power of the interband transition energy in the denominator. Even, so, they do not simply scale with the gap and their sum which is the total value of $\chi^{(2)}$ can either increase or decrease depending on the degree of compensation between the two contributions which was found 
to depend sensitively on details of the calculations, for example, the "non-ideality" of the parameter $u$. Thus, only a very complicated interplay between these different terms is forming the total value of the nonlinear optical response function. A detailed analysis of these contributions appears to be necessary to understand and predict confidently the expected NLO response for new materials.

\section{ACKNOWLEDGMENTS}

We wish to thank Prof. B. Segall for useful discussions. This work was supported by NSF (DMR95-29376).

* Permanent address: P. N. Lebedev Physical Institute, Russian Academy of Sciences, 117924 Moscow, Russian Federation.

[1] F.K. Hopkins, Laser Focus World 31, 87 (1995).

[2] D.W. Fischer and M.C. Ohmer, J. Appl. Phys. 81, 425 (1997).

[3] D.W. Fischer, M.C. Ohmer, P.G. Schunemann, and T.M. Pollak, J. Appl. Phys. 77, 5942 (1995).

[4] J.L. Shay and J.H. Wernick, Ternary Chalcopyrite Semiconductors: Growth, Electronic Properties, and Application (Pergamon, New York, 1975).

[5] H.M. Hobgood, T. Henningsen, R.N. Thomas, R.H. Hopkins, M.C. Ohmer, W.C. Mitchel, D.W. Fischer, S.M. Hegde, and F. K. Hopkins, J. Appl. Phys. 73, 4030 (1993).

[6] G.D. Boyd, E. Buehler, and F.G. Storz, Appl. Phys. Lett. 18, 301 (1971).

[7] D.W. Fischer, M.C. Ohmer, and J.E. McCrae, J. Appl. Phys. 81, 3579 (1997).

[8] R.L. Byer, H. Kildal, and R.S. Feigelson, Appl. Phys. Lett. 19, 237 (1971).

[9] G.D. Boyd, E. Buehler, F. Storz, and J.H. Wernick, IEEE J. Quantum Electron. QE-8, 419 (1972).

[10] V.G. Dmitriev, G.G. Gurzadyan, and D.N. Nikogosyan, Handbook of Nonlinear Optical Crystals, Springer Series in Optical Sciences, vol. 64 (Springer-Verlag, Berlin and Heidelberg, 1991).

[11] S. K. Kurtz, J. Jerphagnon, and M. M. Choy, in Landolt-Börnstein Numerical Data and Functional Relationships in Science and Technology, New Series, vol. III/11 eds. K.-H. Hellwege and A.M. Hellwege, (Springer, Berlin-Heidelberg, 1979), p. 671.

[12] A. G. Jackson, M. C. Ohmer, and S. R. LeClair, Infrared Physics \& Technology 38, 233 (1997).

[13] J. E. Sipe and E. Ghahramani, Phys. Rev. B 48, 11705 (1993).

[14] C. Aversa and J. E. Sipe, Phys. Rev. B 52, 14636 (1995).

[15] S. N. Rashkeev, W. R. L. Lambrecht, and B. Segall, Phys. Rev. B 57, 3905 (1998).

[16] S. N. Rashkeev, W. R. L. Lambrecht, and B. Segall, Phys. Rev. B 57, 9705 (1998).

[17] S. N. Rashkeev, W. R. L. Lambrecht, and B. Segall, in Nitride Semiconductors, eds. F. A. Ponce, S. P. DenBaars, B. K. Meyer, S. Nakamura, and S. Strite, Mater. Res. Soc. Symp. Proc. 482, (MRS Pittsburgh 1998), p. 857.

[18] O.K.Andersen, Phys. Rev. B 12, 3060 (1975).

[19] O.K. Andersen, O. Jepsen, and M. Šob, in Electronic Band Structure and its Applications, ed. M. Yussouf (Springer, Heidelberg, 1987), p. 1.

[20] J.L.P. Hughes and J.E. Sipe, Phys. Rev. B 53, 10751 (1996).

[21] J.L.P. Hughes, Y. Wang, and J.E. Sipe, Phys. Rev. B 55, 13630 (1997).

[22] D.A. Kleinman, Phys. Rev. 126, 1977 (1962).

[23] L. Hedin and B.I. Lundqvist, J. Phys. C 4, 2064 (1971).

[24] Z.H. Levine and D.C. Allan, Phys. Rev. B 43, 4187 (1991); Phys. Rev. B 44, 12781 (1991); Z.H. Levine, Int. J. Quantum Chem S28, 411 (1991).

[25] J. Chen, Z.H. Levine, and J.W. Wilkins, Phys. Rev. B 50, 11514 (1994); Appl. Phys. Lett. 66, 1129 (1995).

[26] L. Hedin and S. Lundqvist, in Solid State Physics, Advances in Research and Applications, eds. F. Seitz, D. Turnbull, and H. Ehrenreich, (Academic Press, New York 1969), vol. 23, p. 1.

[27] N. E. Christensen, Phys. Rev. B 30, 5753 (1984); M. Alouani, L. Brey, and N. E. Christensen, Phys. Rev. B 37, 1167 (1988).

[28] J. E. Jaffe and A. Zunger, Phys. Rev. B 29, 1882 (1984).

[29] J. E. Jaffe and A. Zunger, Phys. Rev. B 27, 5176 (1983).

[30] A. Zunger and J. E. Jaffe, Phys. Rev. Lett. 51, 662 (1983).

[31] A. G. Petukhov, W. R. L. Lambrecht, and B. Segall, Phys. Rev. B 49, 4549 (1993).

[32] R. W. G. Wyckoff, Crystal Structures (New York, Interscience Publishers, 1963).

[33] A. MacKinnon, in Landolt-Börnstein Numerical Data and Functional Relationships in Science and Technology, New Series, vol. III/17h, ed. O. Madelung, (Springer, Berlin-Heidelberg, 1985), p. 9. 
[34] R. Bendorius, V. D. Prochukhan, and A. Sileika, Phys. Stat. Sol. (b) 53, 745 (1972).

[35] S. Limpijumnong, W. R. L. Lambrecht, and B. Segall, unpublished.

[36] E. L. Shirley, X. Zu and S. G. Louie, Phys. Rev. Lett. 69, 2955 (1992).

[37] X. Zhu and S. G. Louie, Phys. Rev. B 43, 14142 (1991).

[38] R. W. Godby, M. Schlüter, and L. J. Sham, Phys. Rev. B 37, 10159 (1988).

[39] O. Zakharov, A. Rubio, X. Blase, M. L. Cohen, and S. G. Louie, Phys. Rev. B 50, 10780 (1994).

[40] J. E. Jaffe and A. Zunger, Phys. Rev. B 30, 741, (1984).

[41] D. A. Roberts, IEEE J. Quantum Electronics 28, 2057 (1992).

[42] C. Sirtori, F. Capasso, D. Sivco, S. Chu, and A. Cho, Appl. Phys. Lett. 59, 2302 (1991).

[43] C. Sirtori, F. Capasso, D. Sivco, A. Hutchinson, and A. Cho, Appl. Phys. Lett. 60, 151 (1992).

[44] R. Atanasov, P. Bassani, and V. M. Agranovich, Phys. Rev. B 50, 7809 (1994).

[45] L. Tsang and S. -L. Chuang, Phys. Rev. B 42, 5229 (1990); Appl. Phys. Lett. 60, 2543 (1992).

[46] L. C. Lew Yan Voon and L. R. Ram-Mohan, Phys. Rev. B 50, 14421 (1994). 
TABLE I. Experimental values of lattice parameters $a, \eta$, and $u$ used in the present calculations.

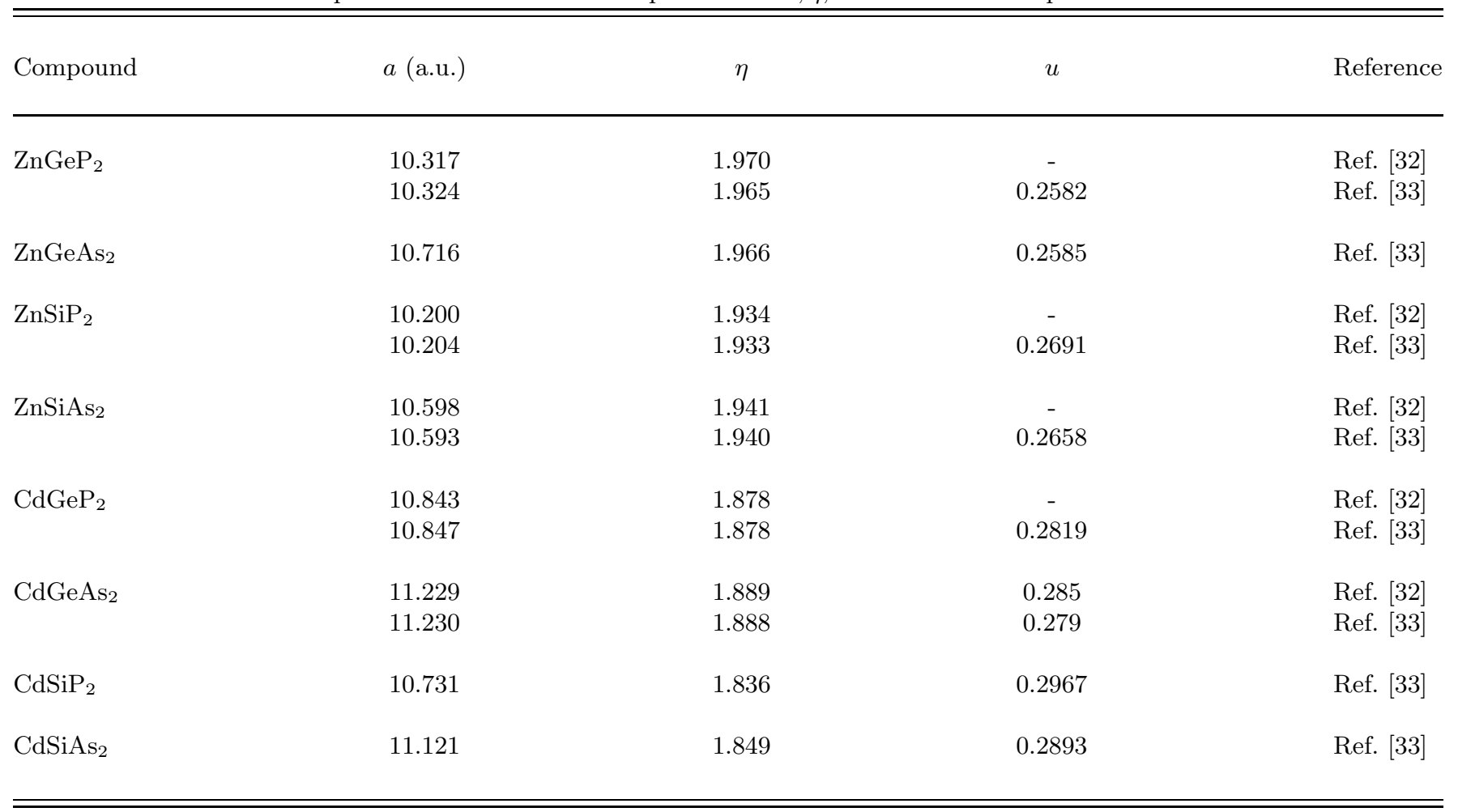

TABLE II. Experimental (Ref. [33]) and LDA values of the energy gap, $\left(E_{g}^{\text {expt }}\right.$ and $\left.E_{g}^{L D A}\right)$, their difference $(\Delta)$, calculated and measured values of the static $\chi^{(2)}$ and its decomposition in inter- and intraband contributions for different ternary semiconductors $\mathrm{ABC}_{2}$ (in pm/V). The values of $\chi^{(2)}$ are compared with experimental data from Refs. [10] and [1]. We use the symbols (d), (pd) and (i) for direct, pseudodirect and indirect gaps respectively.

\begin{tabular}{|c|c|c|c|c|c|c|c|c|}
\hline Compound & $E_{g}^{e x p t}(\mathrm{eV})$ & $E_{g}^{L D A}(\mathrm{eV})$ & $\Delta(\mathrm{eV})$ & $\chi_{\text {total }}^{(2)}$ & $\chi_{\text {inter }}^{(2)}$ & $\chi_{i n t r a}^{(2)}$ & $\chi^{(2)}$ (Ref. 111) & $\chi^{(2)}$ (Ref. 10 ) \\
\hline $\mathrm{ZnGeP}_{2}$ & $2.05(\mathrm{pd})$ & 1.16 (i) & 0.89 & 102 & -231 & 333 & 111 & 150 \\
\hline $\mathrm{ZnGeAs}_{2}$ & $1.15(\mathrm{~d})$ & $0.13(\mathrm{~d})$ & 1.02 & 185 & -328 & 513 & - & - \\
\hline $\mathrm{ZnSiP}_{2}$ & $2.07(\mathrm{pd})$ & $1.22(\mathrm{pd})$ & 0.85 & 61 & -220 & 281 & - & - \\
\hline $\mathrm{ZnSiAs}_{2}$ & $1.74(\mathrm{pd})$ & $0.91(\mathrm{~d})$ & 0.83 & 105 & -210 & 315 & 109 & 146 \\
\hline $\mathrm{CdGeP}_{2}$ & $1.72(\mathrm{~d})$ & $0.75(\mathrm{~d})$ & 0.97 & 127 & -151 & 278 & 162 & 218 \\
\hline $\mathrm{CdGeAs}_{2}$ & $0.57(\mathrm{~d})$ & $-0.44(\mathrm{~d})$ & 1.01 & 506 & -2 & 508 & 351 & 472 \\
\hline $\mathrm{CdSiP}_{2}$ & $2.2-2.45(\mathrm{pd})$ & $1.19(\mathrm{pd})$ & 1.01 & 73 & -139 & 212 & - & - \\
\hline $\mathrm{CdSiAs}_{2}$ & $1.55(\mathrm{~d})$ & $0.42(\mathrm{~d})$ & 1.13 & 139 & -21 & 159 & - & - \\
\hline
\end{tabular}


FIG. 1. Calculated $\varepsilon_{2}(\omega)$ for $\mathrm{ZnGeP}_{2}$ and $\mathrm{CdGeAs}_{2}$ (shifted down for convenience). The solid line corresponds to the polarization $\mathbf{E} \perp \mathbf{c}$, the dotted line corresponds to $\mathbf{E} \| \mathbf{c}$.

FIG. 2. The calculated $\Delta n$ for $\mathrm{ZnGeP}_{2}$ (solid line) and $\mathrm{CdGeAs}_{2}$ (dotted line) in a wide energy region.

FIG. 3. Calculated birefringence for $\mathrm{ZnGeP}_{2}$ (solid line) together with experimental data of Ref. [3] at room temperature. The open circles and filled circles correspond to measurements on two different $\mathrm{ZnGeP}_{2}$ samples.

FIG. 4. Calculated birefringence for CdGeAs 2 (solid line) together with room temperature measurements of Ref. [7] (filled circles).

FIG. 5. Calculated 123 (solid line) and 312 (dotted line) components of the imaginary part of SHG for ZnGeP 2 .

FIG. 6. Calculated 123 (solid line) and 312 (dotted line) components of the imaginary part of SHG for CdGeAss.

FIG. 7. Calculated interband (solid line) and intraband (dotted line) contributions to $\operatorname{Im} \chi^{(2)}(\omega)$ for $\mathrm{ZnGeP}_{2}$ (123 component).

FIG. 8. Calculated interband (solid line) and intraband (dotted line) contributions to $\operatorname{Im} \chi^{(2)}(\omega)$ for CdGeAss $(123$ component).

FIG. 9. Electronic bands of $\mathrm{ZnGeP}_{2}$ along the $Z-\Gamma$ and $\Gamma-X$ lines in the Brillouin zone $(Z=2 \pi / \eta a \cdot(0,0,1) ; \Gamma=(0,0,0)$; $X=2 \pi / a \cdot(1 / 2,1 / 2,0))$ for the experimental crystal structure (solid lines) and for the ideal chalcopyrite structure $(u=1 / 4$, $\eta=2$ ) with the same lattice constant $a$ (dotted lines). Both the structures are shown in LDA (without gap corrections). The energy in both cases is counted from the top of the valence band.

FIG. 10. The LDA electronic bands of $\mathrm{CdSiP}_{2}$ for the experimental crystal structure $(\eta=1.836, u=0.2967$, solid lines $)$ compared with bands for the structure with the same $a$ and $\eta$ but with the value of $u$ for the ideal chalcopyrite structure $(u=1 / 4$, dashed lines).

FIG. 11. The LDA electronic bands of $\mathrm{CdSiP}_{2}$ for the crystal structure with $(\eta=2, u=1 / 4$, solid lines $)$ compared with bands for the ideal chalcopyrite structure $(\eta=2, u=1 / 4$, dashed lines) with the same lattice constant $a$.

FIG. 12. The LDA electronic bands for the $1+1$ alternative $\mathrm{ZnGeP}_{2}$ structure. 


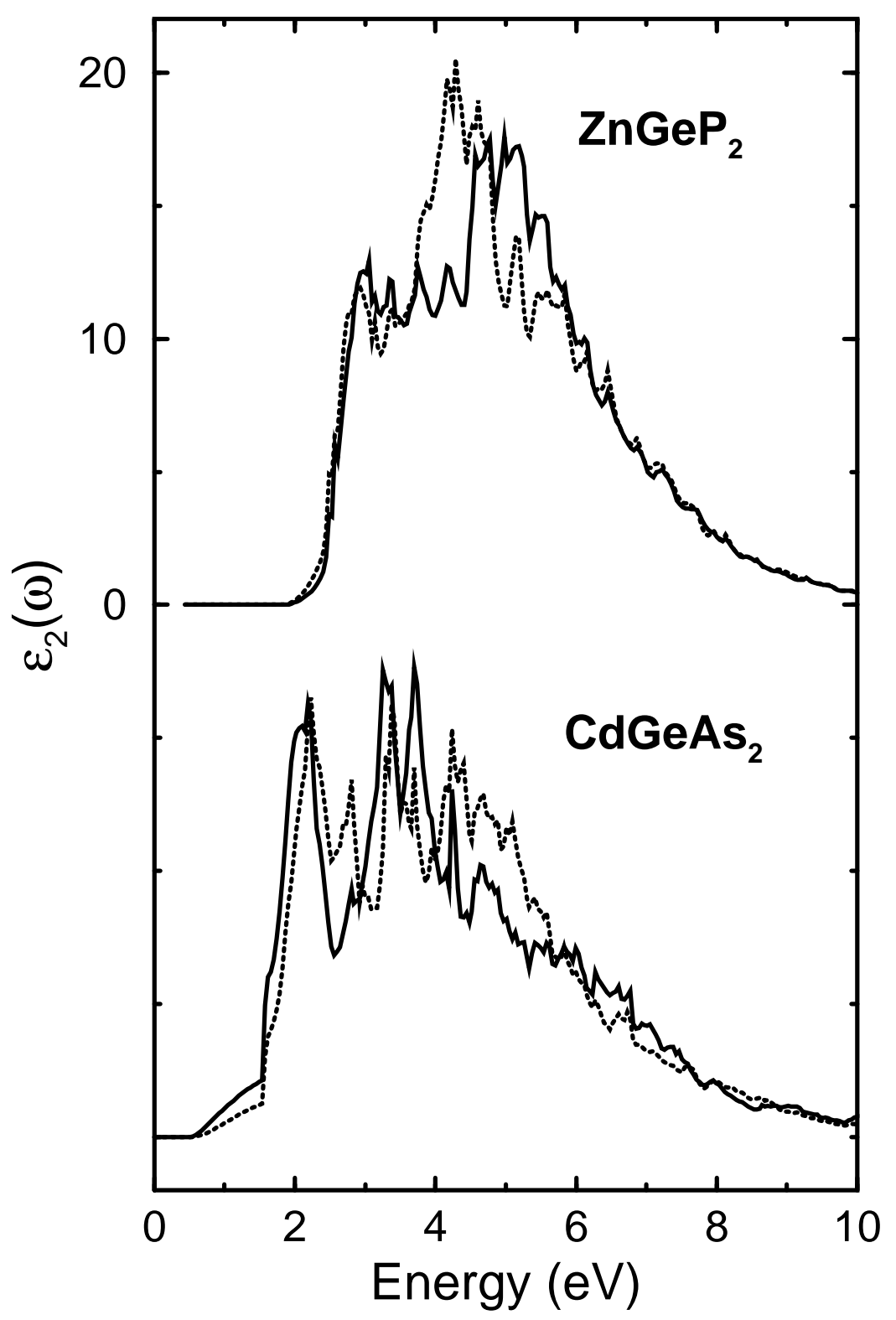




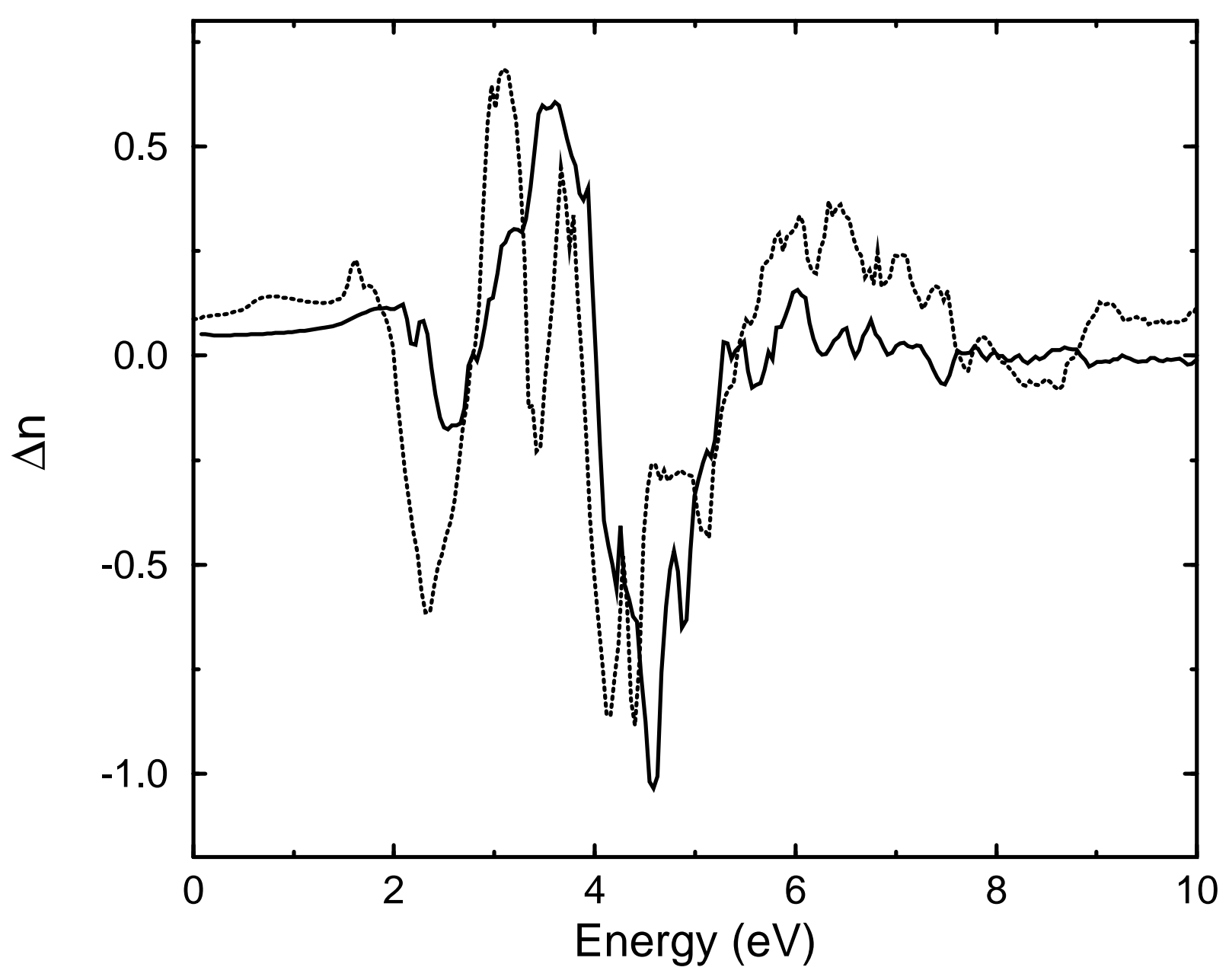




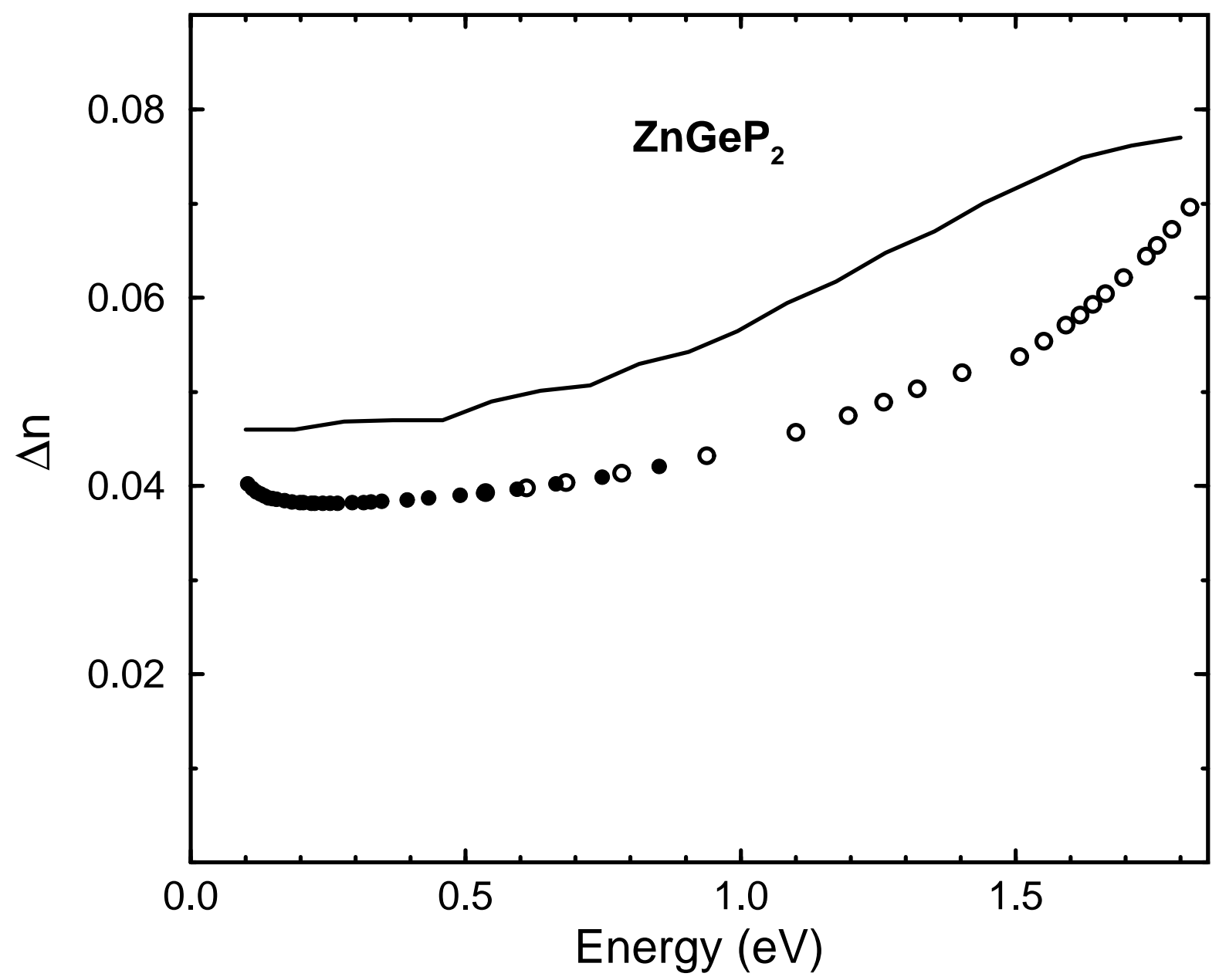




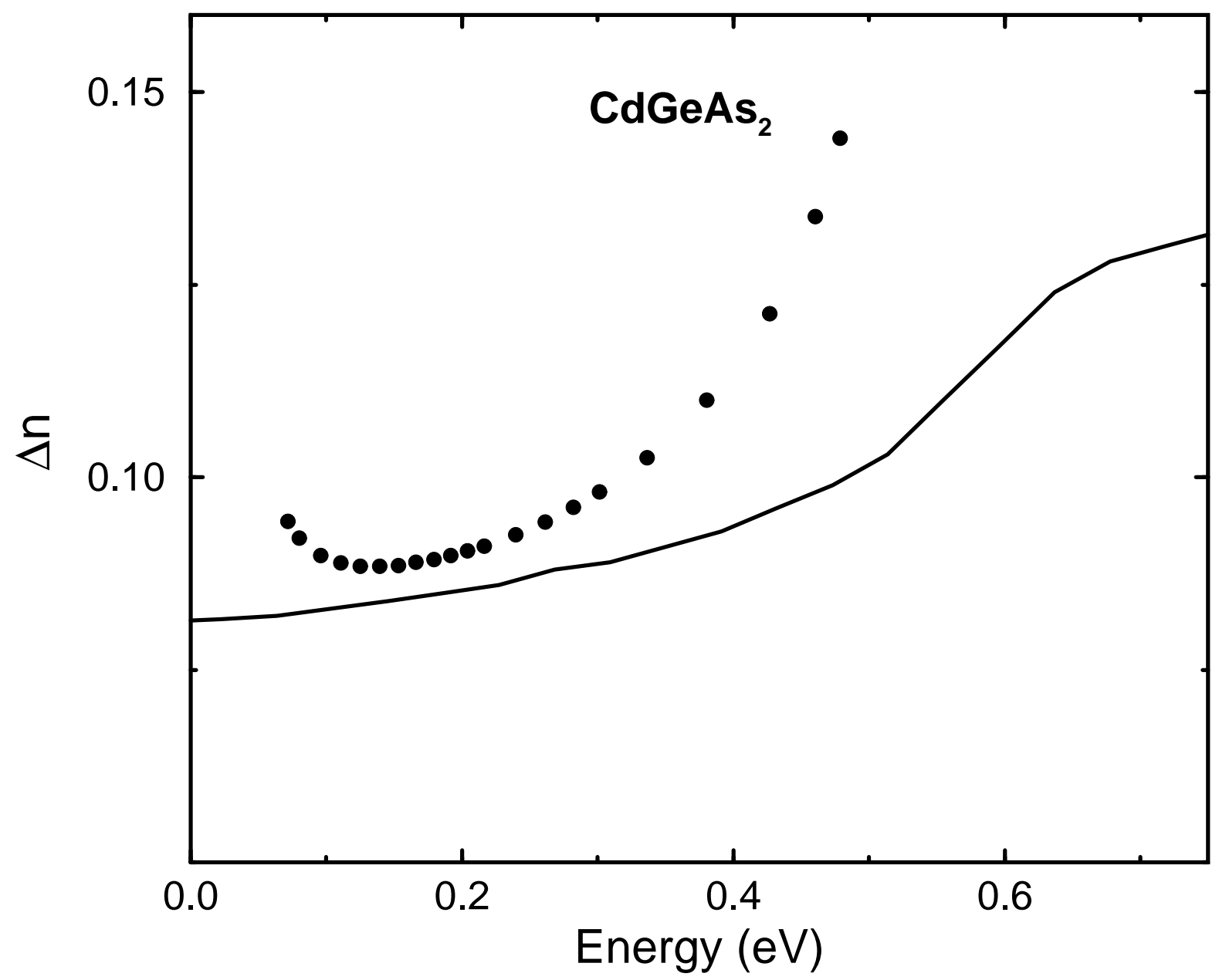




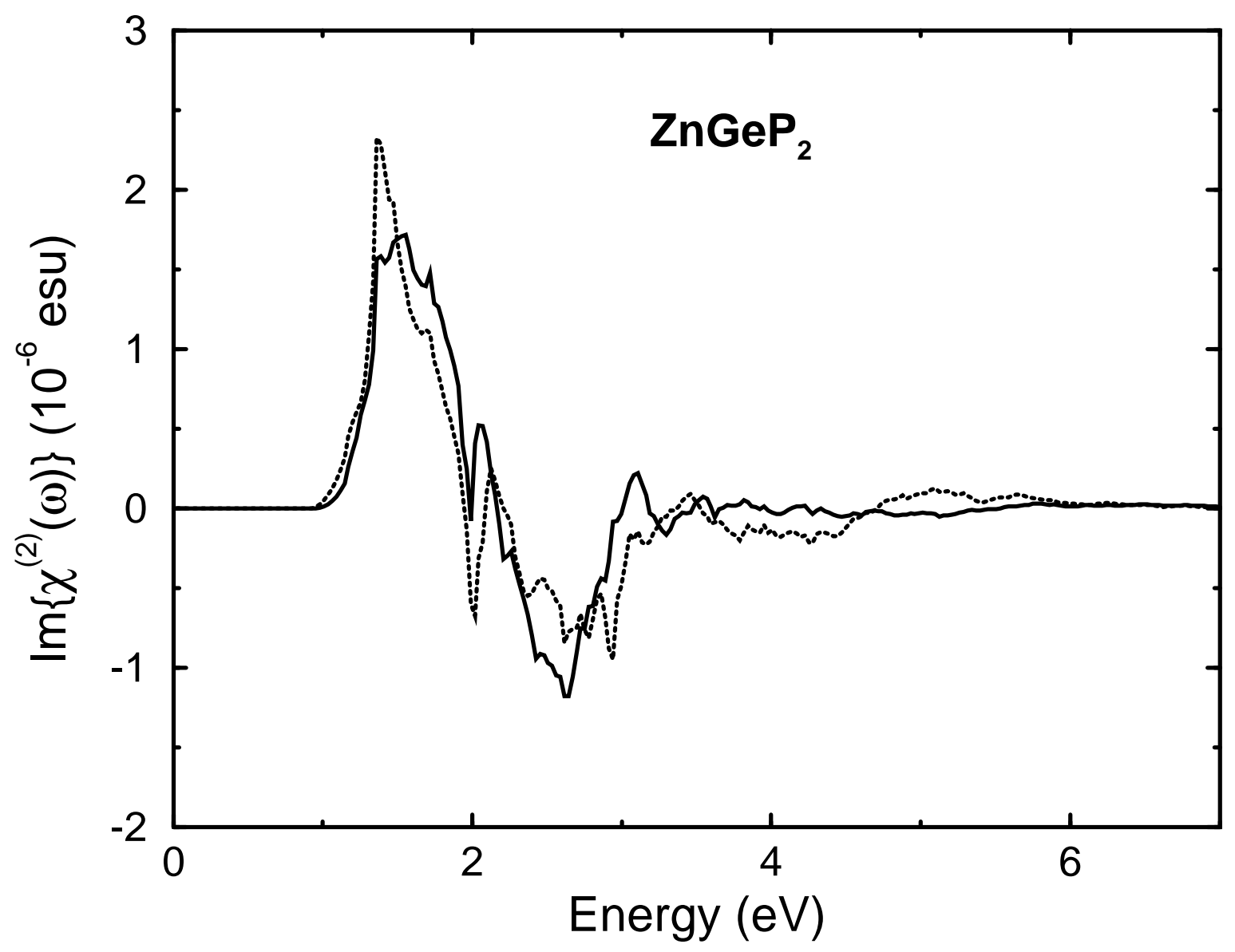




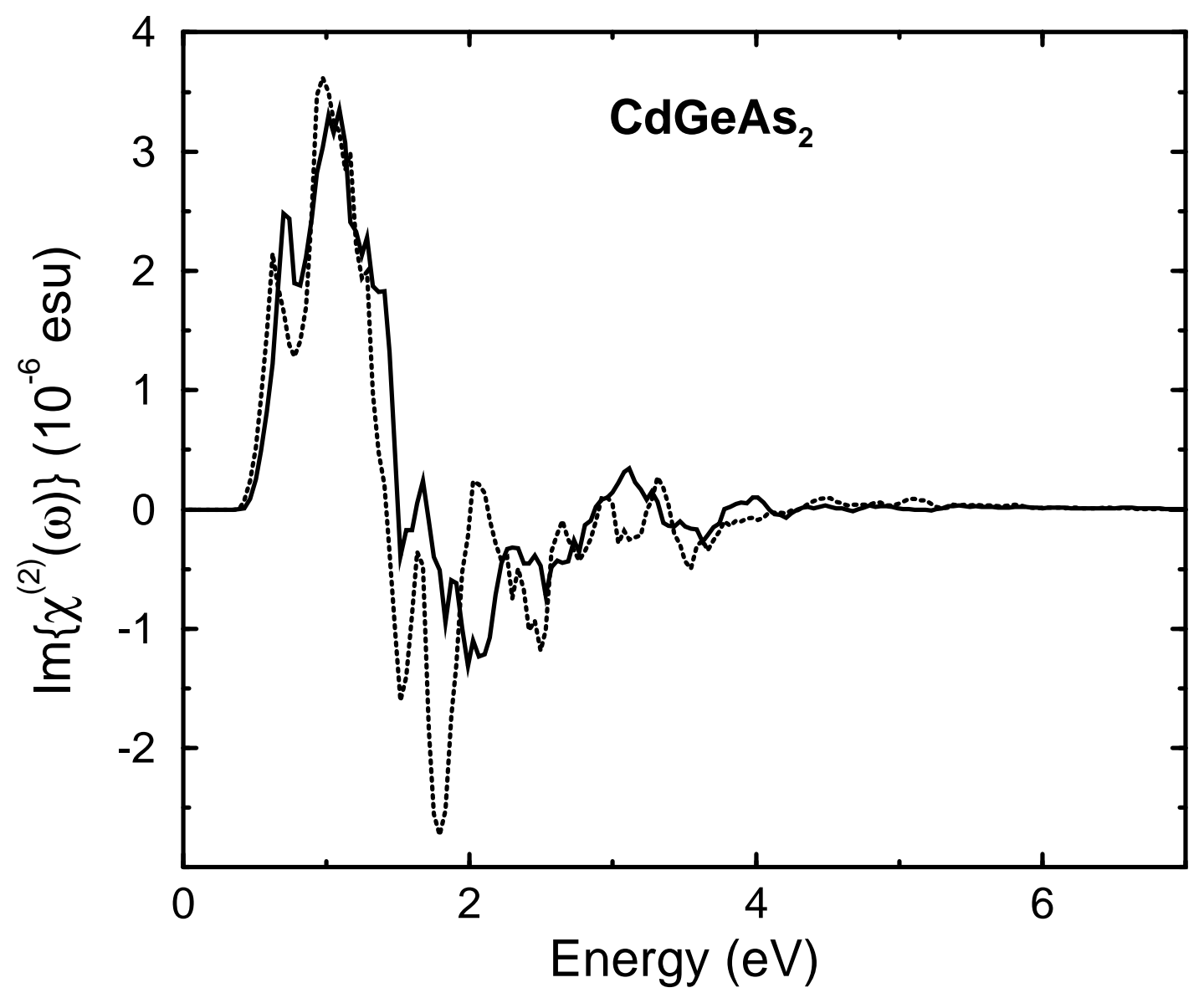




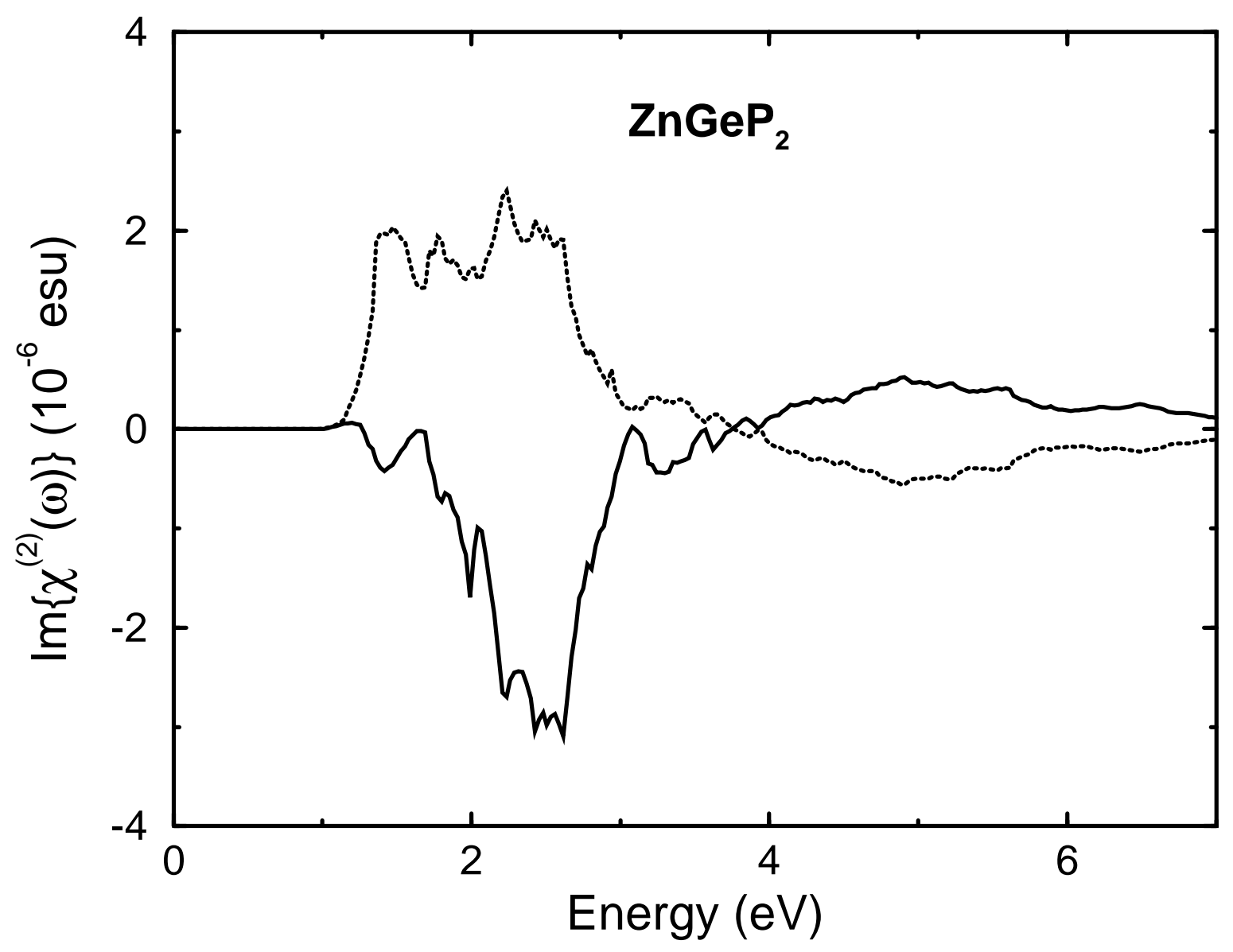




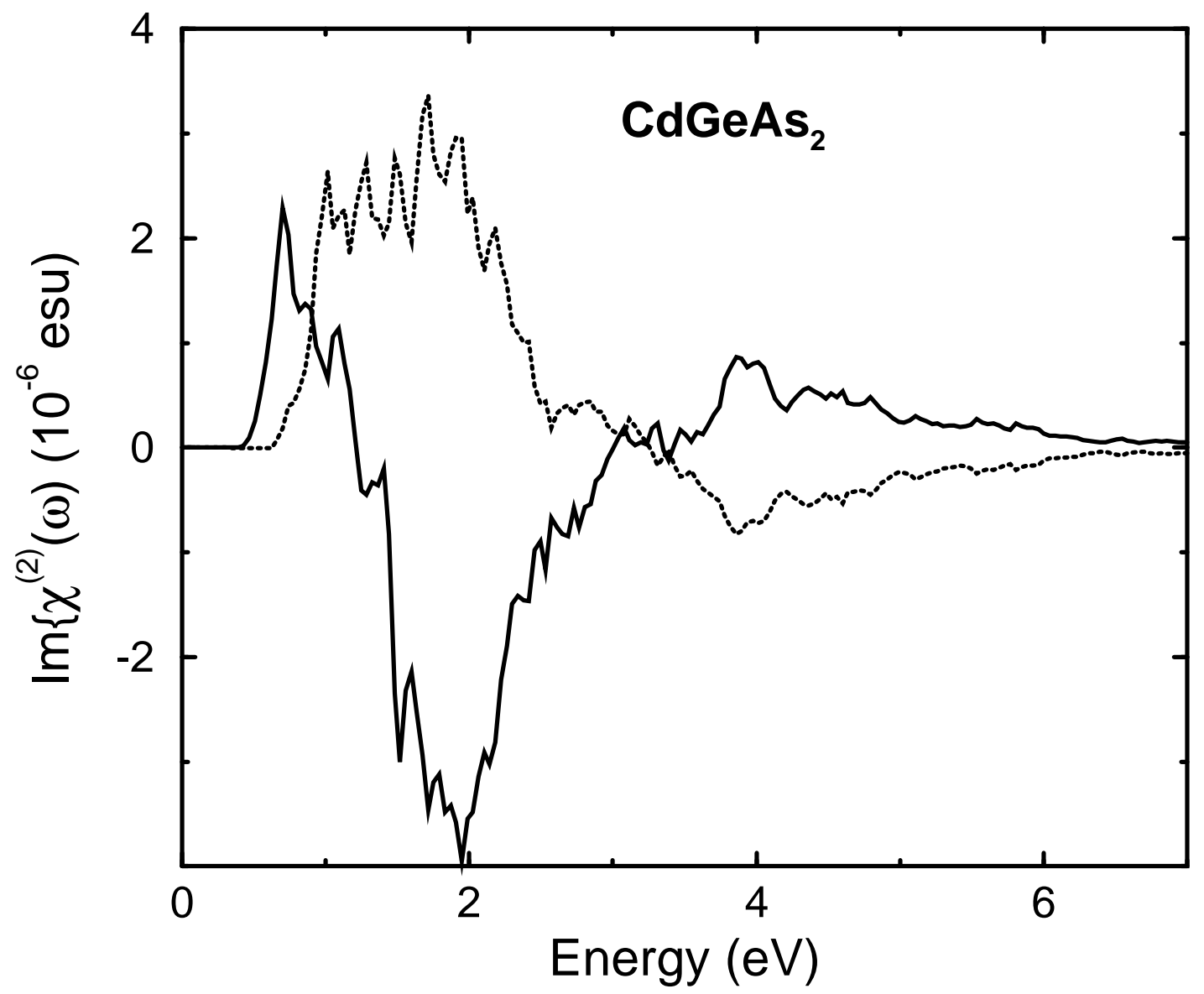




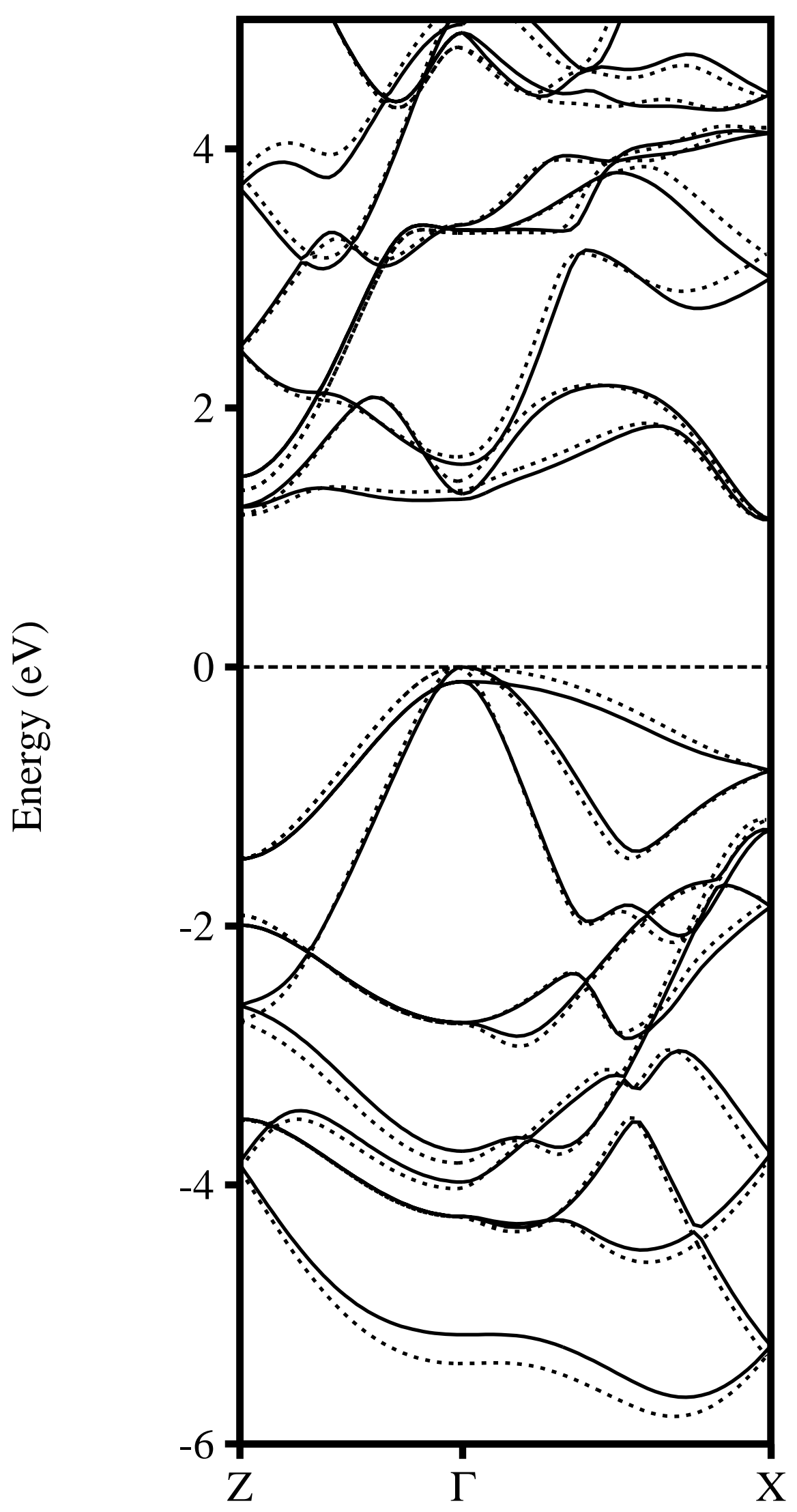




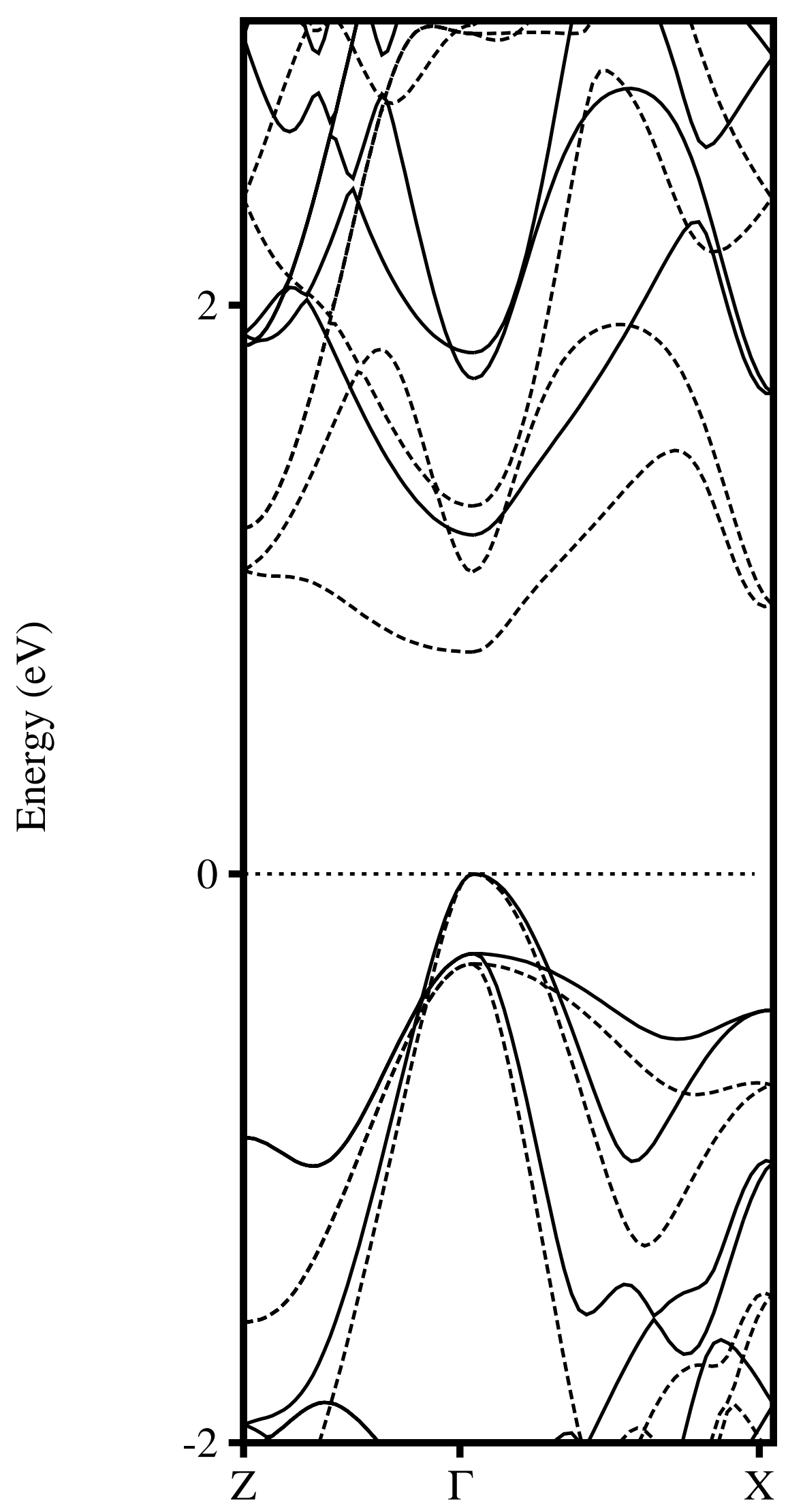




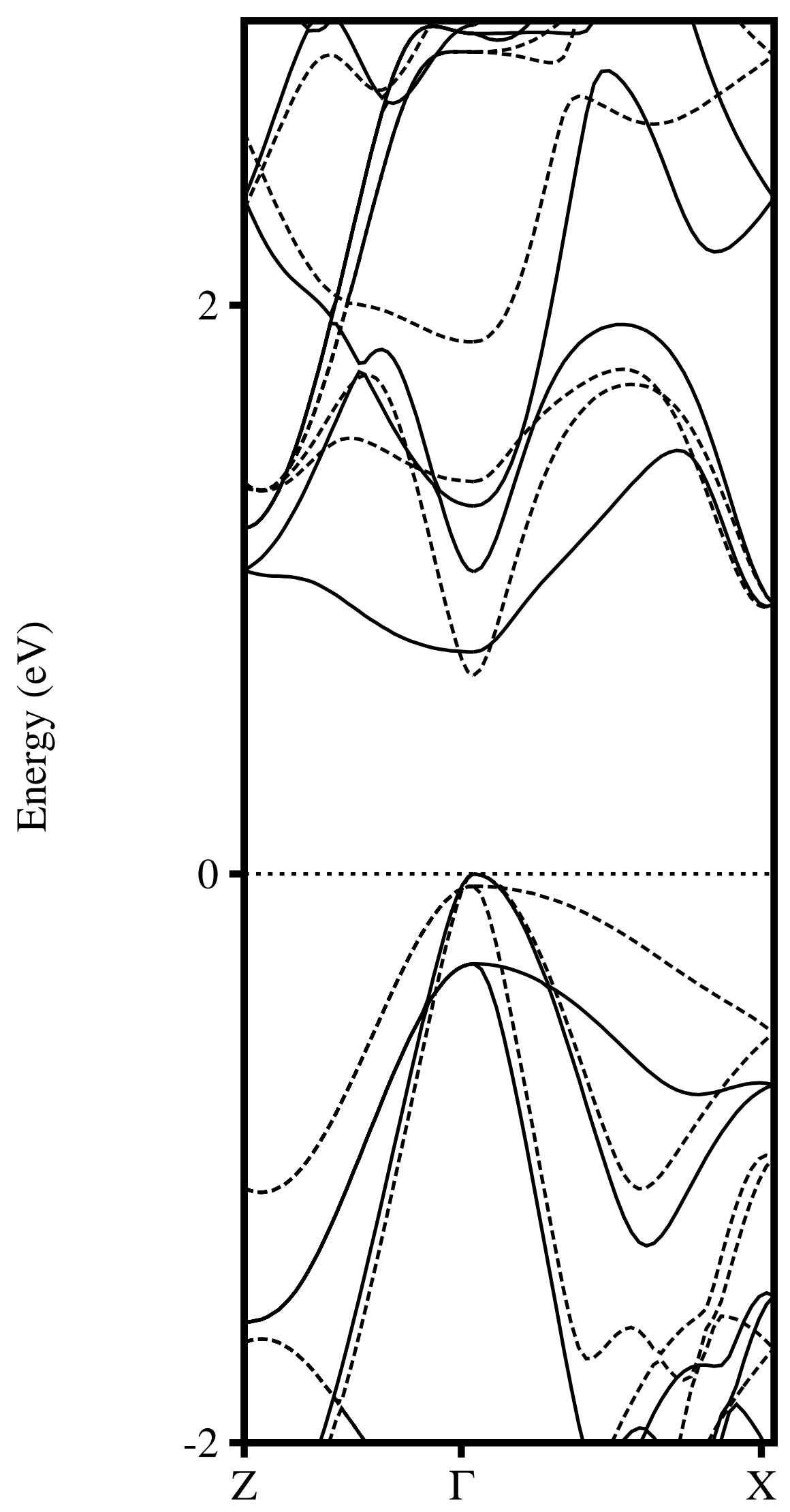




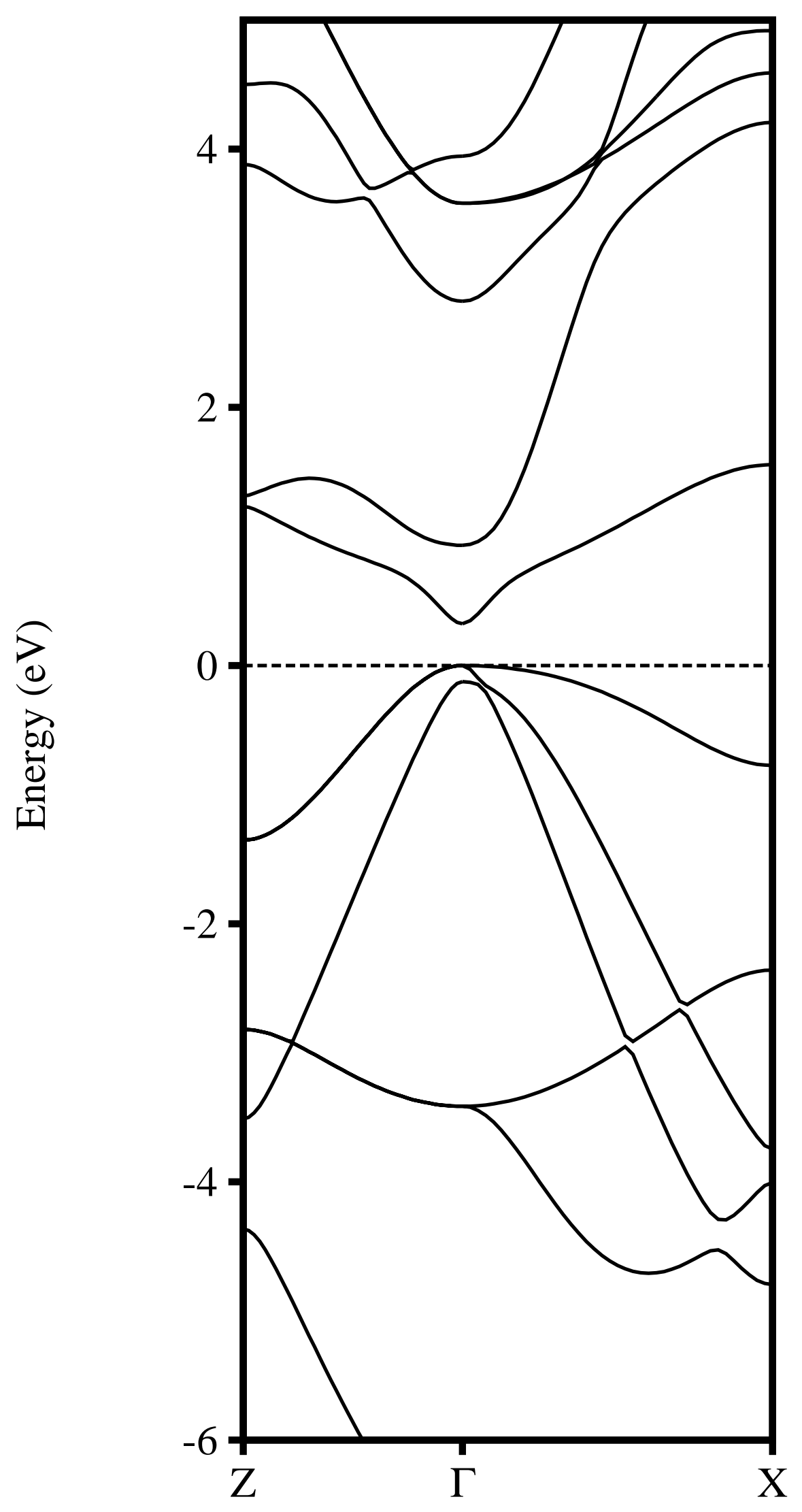

